\title{
applied sciences
}

ISSN 2076-3417

www.mdpi.com/journal/applsci

Review

\section{Optical Current Sensors for High Power Systems: A Review}

Ricardo M. Silva ${ }^{1}$, Hugo Martins ${ }^{1,2}$, Ivo Nascimento ${ }^{1,2}$, José M. Baptista ${ }^{1,3}$, António Lobo Ribeiro ${ }^{4}$, José L. Santos ${ }^{1,2}$, Pedro Jorge ${ }^{1}$ and Orlando Frazão ${ }^{1, *}$

1 INESC Porto, Rua do Campo Alegre, 687, 4169-007 Porto, Portugal;

E-Mails: rmsilva@inescporto.pt (R.M.S.); hfm@inescporto.pt (H.M.); pjorge@inescporto.pt (P.J.)

2 Dept Física e Astronomia da faculdade de Ciências da Universidade do Porto, Rua do Campo Alegre, 687, 4169-007 Porto, Portugal; E-Mail: ivomac88@gmail.com (I.N.); josantos@fc.up.pt (J.L.S.)

3 Centro de Competência de Ciências Exatas e da Engenharia, Universidade da Madeira, Campus da Penteada, 9000-390 Funchal, Portugal; E-Mail: jmb@uma.pt

4 Faculty of Health Sciences, University Fernando Pessoa, R. Carlos da Maia 296, 4200-150 Porto, Portugal; E-Mail: alobo@ufp.edu.pt

* Author to whom correspondence should be addressed; E-Mail: ofrazao@inescporto.pt; Tel.: +351-220-402-301; Fax: +351-220-402-734.

Received: 23 May 2012; in revised form: 15 June 2012 / Accepted: 18 June 2012 /

Published: 2 July 2012

Abstract: The intrinsic advantages of optical sensor technology are very appealing for high voltage applications and can become a valuable asset in a new generation of smart grids. In this paper the authors present a review of optical sensors technologies for electrical current metering in high voltage applications. A brief historical overview is given together with a more detailed focus on recent developments. Technologies addressed include all fiber sensors, bulk magneto-optical sensors, piezoelectric transducers, magnetic force sensors and hybrid sensors. The physical principles and main advantages and disadvantages are discussed. Configurations and strategies to overcome common problems, such as interference from external currents and magnetic fields induced linear birefringence and others are discussed. The state-of-the-art is presented including commercial available systems.

Keywords: optical current sensors; magnetooptic current sensors; fiber optic current sensors; Faraday effect 


\section{Introduction}

Due to the massive growth in the use of electric equipment in developed countries and the worldwide increase of the electrical distribution/consumption, the interest in sensors for electrical current metering, particularly in high voltage levels, has increased significantly over the last decade. Although optical current sensors are still too expensive for low voltage applications (such as, in residential areas), they are extremely attractive for high voltage applications (such as, in electrical high voltage (EHV) substations) when compared with conventional sensors, due to their intrinsic properties, such as:

- Immunity against electromagnetic interferences (EMI);

- Electrical isolation (the optical sensors are made of dielectric materials);

- Possibility for measuring AC and DC;

- Absence of saturation effects;

- Low power consumption;

- Small size, lightweight, and relatively low cost.

Furthermore they are usually connected to optical fibers that have large communication bandwidths, and due to their very low absorption loss allow remote detection, high multiplexing capability and data transmission over long distances. Also, optical current sensors measure the magnetic field generated by the electric current rather than the current itself, thus avoiding the electric hazards that the high voltage measurements imply.

There are basically two linear effects by which the magnetic field can be measured by optical sensors: magneto-optic effect (or Faraday effect) and magnetic force (or Lorentz force).

In the case of sensors that use the Faraday effect, a rotation in the angle of polarization of the light propagating in magneto-optic material is induced by an external magnetic field. A short explanation regarding the Faraday effect, as well as the different interrogation techniques that can be used to interrogate the polarization rotation angle, are presented. Then, two different groups of optical current sensors using this effect (all-fiber optic sensors and bulk-optic based sensors) are analyzed. A key aspect of the concept of all-fiber optic sensors is that the optical fiber acts both as the sensing element and communication channel, which allows for simple solutions and reduces the losses in the fiber connections. By winding the optical fiber around the electric conductor, immunity to external currents or magnetic fields [1], as well as, tunable sensitivity can be easily achieved. As for bulk-optic sensors, they usually present higher sensitivity and robustness, which are very important aspects in real applications. In both cases, saturation effects such as the ones that occur in ferromagnetic-based sensors are avoided allowing higher measurement ranges.

As for the magnetic force sensors, they measure the force applied by the magnetic field on the sensing element. The possibility of bonding or jacketing optical fibers with magnetostrictive materials to measure the intensity of the magnetic field is also discussed, with special emphasis to Terfenol-D, which has the highest magnetostriction of any alloy. With these types of sensors, multi-point magnetic field measurements are possible with a single optical fiber (optical multiplexing). Other solutions using magnetic fluids are also discussed.

Most optical sensors described can be installed without the interruption of the current in the conductor, which is a very advantageous property when dealing with high voltage distribution systems. 
Lastly, the possibility of hybrid solutions for current measurement, using both conventional technology and new (optical) technology, are also discussed. Besides some operational advantages, these hybrid sensors may also be very important in the first step of implementing optical current sensors in the industry, before developing fully optical current measuring systems.

\section{Faraday Effect}

In 1845, Michael Faraday discovered a difference in refractive index of glass for left-handed and right-handed circularly polarized light induced by an external magnetic field. Émile Verdet showed in 1854 , that the angle of rotation of linearly polarized light is proportional to the strength of the magnetic field and the cosine of the angle between the field and the propagation direction of the light wave. This rotation can be expressed mathematically by:

$$
\theta_{f}=\int_{L} V \vec{B} \cdot \overrightarrow{d l}
$$

where, $V$ is the material Verdet constant, which is both dispersive and temperature-dependent $[1,2]$, $B$ is the magnetic flux density vector and $d l$ is the differential vector along the direction of propagation. This effect is called the Faraday effect or linear magneto-optic effect and can be used to build optical current sensors. Figure 1 illustrates the polarization rotation due to a parallel external magnetic field on a magneto-optical material, such as, glass.

Figure 1. Faraday effect in linearly polarized light.

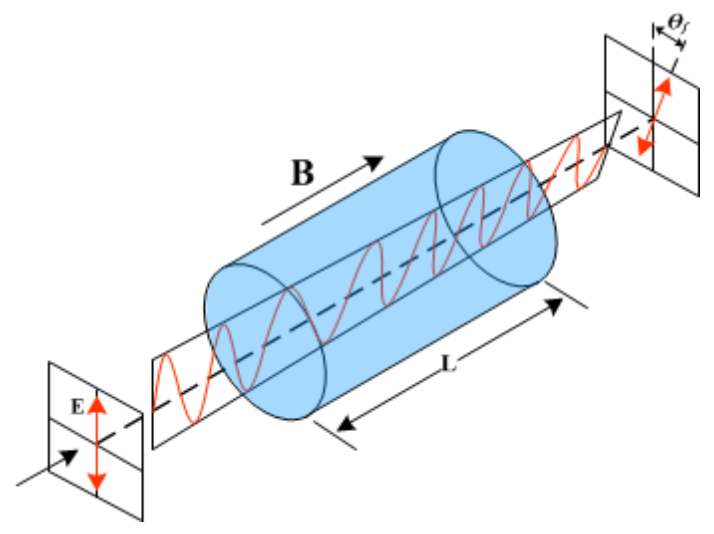

The Faraday effect is distinct from the inherent circular birefringence (called optical activity) that appears in some materials, in that its sign depends on the direction of the magnetic field with the respect to the direction of propagation of the light [3]. That is to say, it is non-reciprocal. Therefore, if the same light passes through the same medium, but with opposite propagation direction, the rotation of polarization will be cumulative

The Faraday effect is present, to some extent, in all materials and its characteristics are related to the other magnetic properties of the material. It varies with temperature, much more in paramagnetic and ferromagnetic than in diamagnetic materials. It is linear with magnetic field in diamagnetic materials but saturates in ferromagnetic materials, and its magnitude generally diminishes with increasing wavelength. 


\section{Interrogation Techniques}

In order to measure the Faraday rotation of the azimuth output light there are two main detection schemes that can be employed: polarimetric and interferometric.

\subsection{Polarimetric Detection}

\subsubsection{Basic Polarimetric Scheme}

Different signal analysis techniques can be used to quantify the Faraday rotation of the azimuth of the output light from an optical current sensor. One way to detect this rotation is by using a polarimetric detection scheme, which consists of two polarizers, one at the input of the sensor and the other at the output, arranged as shown schematically in Figure 2. The first polarizer has the objective of defining the initial polarization state of the light wave and the second polarizer is used to adjust the sensor sensitivity and transforms the polarization rotation into a light intensity modulation that can be measured using a photodetector [1].

Figure 2. Polarimetric detection scheme.

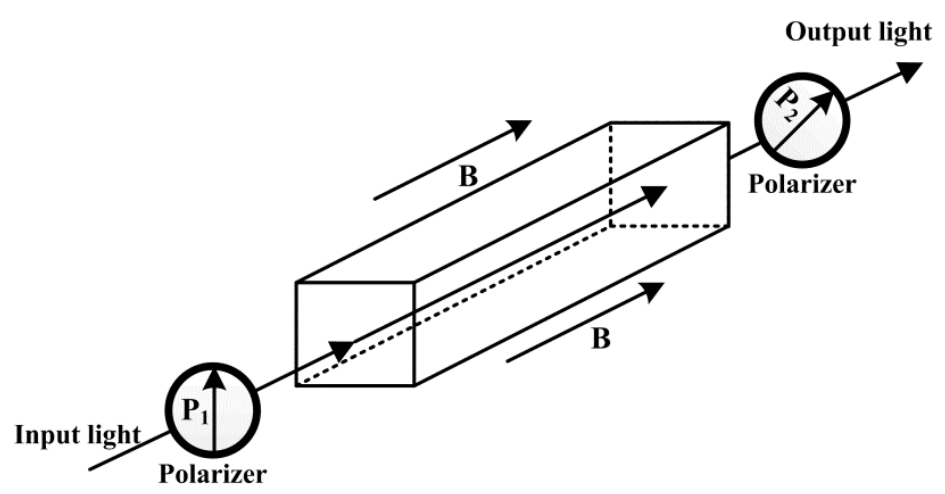

According to Malus law, when two polarizers are in such arrangement, the intensity of the output light is given by [2]:

$$
I_{\text {out }}=I_{P I} \cdot \cos ^{2}(\alpha)
$$

where $I_{P 1}$ is the light intensity after the first polarizer and $\alpha$ is the relative angle between the transmission axes of the polarizers. As we can see from Figure 3, maximum sensitivity is achieved when this angle is $45^{\circ}$. This means that with the polarizers having a relative angle of $45^{\circ}$, any small change in the plane of polarization of the light, in the path between the two polarizer's, induced by an external magnetic field, will be transformed in a larger change of intensity at the output. 
Figure 3. Transfer function of two polarizers showing the transmitted power as a function of their relative angle.

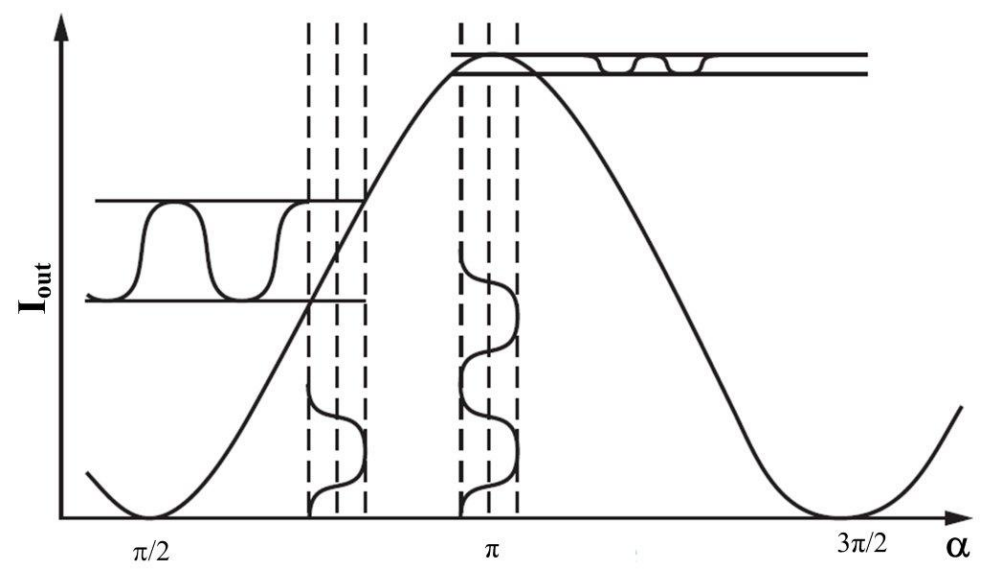

Adjusting the polarizers for the maximum sensitivity, the sensor transfer function is [1]:

$$
I_{\text {out }}=\frac{I_{\text {in }}}{2}\left[1+\sin \left(2 \theta_{f}\right)\right]
$$

In order to eliminate the dependence of the sensor response to the input light intensity fluctuations, the final processed output signal can be obtained by dividing the AC component by the DC component [1]. That is:

$$
S_{A C}=\sin \left(2 \theta_{f}\right)
$$

\subsubsection{Dual Quadrature Scheme}

Another improvement of the processing scheme presented above is shown in Figure 4. In this case, the output light is divided into two orthogonal polarizations, through the use of a Wollaston prism, and these two signals are detected by two independent photodetectors and processed by an analog circuit that computes the output signal $S$, given by Equation (4).

Figure 4. Schematic of the dual-quadrature polarimetric detection incorporating optical fiber links. Legend: GRIN (GRadient INdex); $\mathrm{P}_{\mathrm{D}}$ (Photodetector); $\mathrm{P}_{i}(I=\mathrm{x}, \mathrm{y})$ optical power in polarizations states.

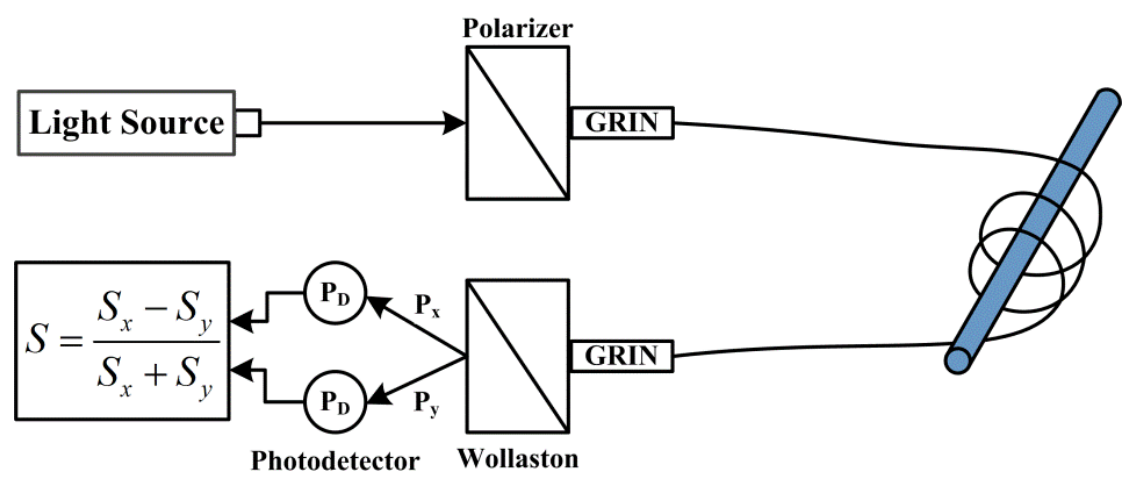

Instead of using a Wollaston prism, it is possible to use a fiber coupler to divide the signal into two polarized states at $\pm 45^{\circ}$ with respect to the input polarizer, and allowing the generation of two output 
signals with opposite phase. This dual-quadrature scheme offers better common noise rejection than the previous one.

When the Faraday rotation is relatively small and we include the linear birefringence $(\beta)$ effect in the sensing element, Equation (4) becomes:

$$
\begin{array}{cc}
S_{A C}=2 \theta_{F} \frac{\sin \beta}{\beta} & \beta \gg 2 \theta_{F} \\
S_{A C}=2 \theta_{F} & \beta \ll 2 \theta_{F}
\end{array}
$$

The two methods presented above do not take into account the linear birefringence effect, which as can be seen from Equation (5), can greatly reduce the sensitivity of the sensor. Furthermore, because the linear birefringence $(\beta)$ is usually temperature dependent, severe measurement errors can be introduced. To account for linear birefringence, it is necessary to introduce other detection methods that require more complex signal processing strategies that will be described in the following section [1].

\subsection{Interferometric Detection Schemes}

Previously in the polarimetric detection scheme, the rotation of the plane of polarization of linear polarized light was analyzed. This rotation can also be analyzed in terms of circular polarization, corresponding to a phase difference between the two circular orthogonal modes (left-handed and right-handed circular polarization). This can be done using an interferometric detection scheme where a modulation frequency carrier is generated, and the optical phase variation that is modulated by time delay induced between arms of the interferometer, will contain the electric current information. This phase carrier can be generated by using an unbalanced Michelson or Mach-Zehnder interferometer, or other interferometric configuration, such as Sagnac interferometer [1,3].

Figure 5 shows schematically a current sensing Sagnac interferometer, which is commonly used in gyroscopes and it is sensitive to non-reciprocal effects [4]. This interferometer is interrogated by using a heterodyne detection scheme.

Figure 5. Sagnac loop interferometer current sensor.

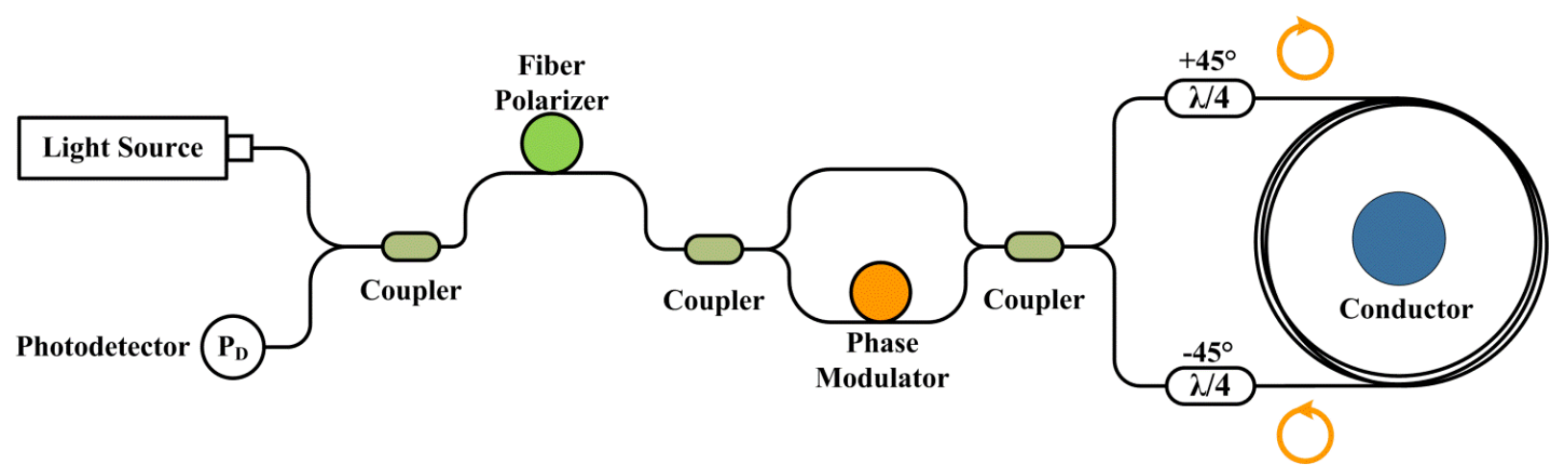

In this particular application, light from an optical broadband source, is linearly polarized with a fiber polarizer and is injected into the Sagnac loop with crossed $\lambda / 4$-wave plates mounted at an angle of $45^{\circ}$ and $-45^{\circ}$ relative to the plane of polarization of the linearly polarized inputs, for the upper and lower plate, respectively. In this arrangement each of the counter propagating waves are converted to 
orthogonal circular states. These two counter-propagating waves travel the Sagnac loop with different velocities, due to the external magnetic field induced circular birefringence. After crossing the loop they are converted again into linear polarization modes and interfere after crossing the output linear polarizer. The phase information is retrieved with the introduction of the phase modulator on the unbalanced Mach-Zehnder interferometer, which can be used to generate the phase carrier. Using either Pseudo-Heterodyne or Heterodyne processing schemes, the relative phase accumulated in the Sagnac loop, which is proportional to the magnetic field generated by the electric current on the conductor, can be recovered. Some disadvantages of using this type of interferometer include temperature and vibration dependent sensitivities.

An improved version of the above system can be done in a reflection configuration, as shown in Figure 6.

Figure 6. In-line interferometer current sensor.

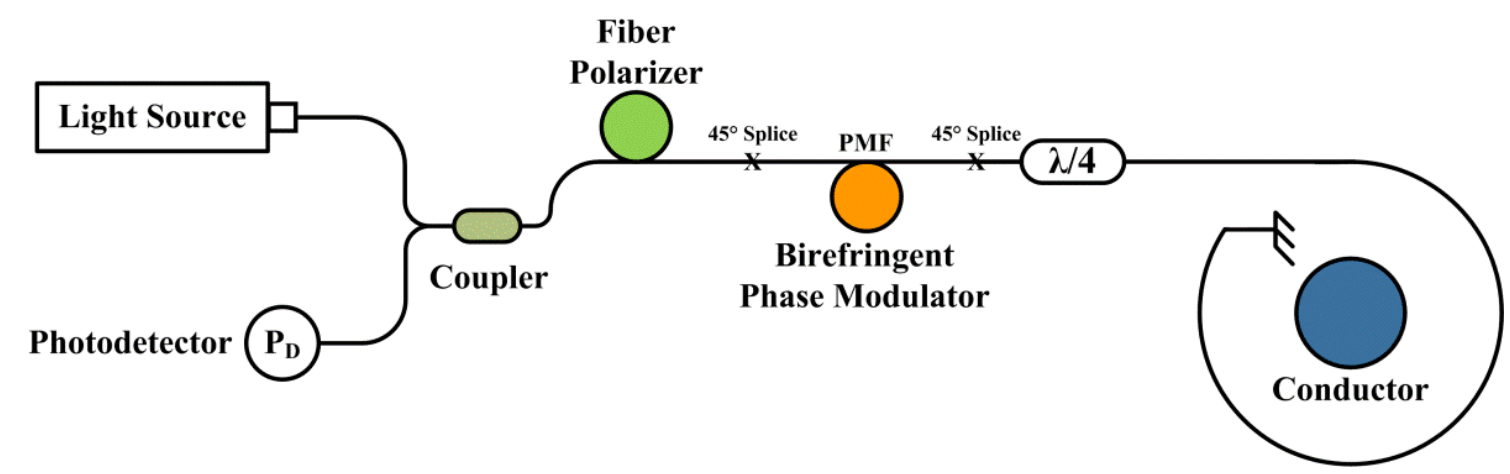

Between the optical source and the $\lambda / 4$-wave plate, the polarized light is injected at $45^{\circ}$ into a polarization maintaining fiber (PMF). After crossing the PMF, the $\lambda / 4$-wave plate which is oriented at $45^{\circ}$ with respect to the birefringence axis of the PMF, transforms the two orthogonal polarization modes into circular polarization modes with opposite rotation directions. At the end of the fiber, reflection occurs and the reverse process occurs. Due to reflection configuration, the states of polarization swaps and each polarization component travels the optical path made by the other polarization. At the end, both polarization pass through the same optical path and compensate all reciprocal effects, but still maintaining a phase difference proportional to the electric current to be measured. Due to the operation in reflection this scheme also doubles the sensitivity in relation to the first one, for the same number of fiber turns around the wire.

The modulation introduced in the polarization interferometer (the length of PMF), near the $45^{\circ}$ splice enhances the ability to detect the small phase differences, produced by small currents. Although the two schemes described permit a certain degree of immunity to reciprocal perturbations, timevarying disturbances which alter the birefringence of the PM fiber, affect counter-propagating waves differently and are not compensated [4]. Also, the fact that we are working with interferometers, means that it is necessary to use a stable optical source, and the modulation despite granting certain immunity to low frequency noise, limiting the system bandwidth. Another crucial problem is due to the phase difference introduced by the $\lambda / 4$-wave plate that can have small deviations and therefore change the system sensibility [3]. 


\section{State of Art}

Nowadays there is a great diversity of optical current sensors. Taking into account the sensing mechanism employed and the materials used, optical current sensors can be organized in four main groups $[1,5]$ :

- All-fiber sensor: The fiber itself acts as a transducer mechanism. The magneto-optical effect (or Faraday effect) is used to induce a rotation in the angle of polarization of the light propagating in the fiber, which is proportional to the magnetic field. Usually, the fiber is coiled around the electrical conductor, making it immune to external currents and magnetic fields.

- Bulk optic sensor: These sensors use a piece of glass or crystal with high Verdet constant as the transducer, which is placed near (or around) the electrical conductor. The magnetic field is also measured using the magneto-optical effect. These sensors are usually cheap, robust and more sensitive.

- Magnetic force sensors: In an analogous process to the piezoelectric elements, when a magnetic field is applied to a magnetostrictive element it induces mechanical changes in the material. These changes can be again measured by attaching a fiber Bragg grating (FBG) to the magnetostrictive element.

- Hybrid sensors: These sensors employ some of the standard electromagnetic technology already existent and some of optical technology. In this case, the first current transducer is done with conventional electromagnetic technology (such as, a Rogowski coil) but its interrogation and information transportation is done by an optical fiber system. The objective of these sensors is to construct an interrogation system that takes advantages of the high level of electrical isolation offered by optical fibers and avoids difficulties associated with birefringence.

\subsection{All-Fiber Sensors}

All-fiber sensors use very simple configurations because the fiber can be simply coiled around the electric conductor to be measured. Also, the sensor sensibility can be changed by simply changing the number of turns of the optical fiber around the conductor [3]. Since these kind of devices usually uses several meters of fiber, the sensors are more vulnerable to pressure and temperature gradients, mechanical vibrations and other environmental noises than smaller devices (such as bulk-optic).

When operating at high currents, the magnetic crosstalk between different conductors can also be a relevant problem. Schemes to reduce the magnetic crosstalk in three-phase electric systems have been proposed [6]. However, if the detection scheme forms a closed circuit around the electrical conductor, as most of them do, it will not be sensitive to external magnetic fields.

Typically, standard silica fibers have a low Verdet constant when compared with bulk-optic glasses. Many studies have been done in order to increase the Verdet constant of the fibers.

Materials such as flint glass have been tested and showed a Verdet constant six times higher than fused silica and much lower photo-elastic coefficient (780 times) [3,7,8]. A detailed review of current transducers using flint glass fibers can also be found in the literature [9]. The study provides specific information of the sensors (setup configurations, temperature dependence, accuracy, etc.) and the 
fibers used (Verdet constants, photo-elastic constants, wavelength dependence, etc.) as well as results of field tests performed. In Figure 7, a typical current sensor with flint fiber is represented.

Figure 7. Typical configuration of an optical current sensor with flint fiber.

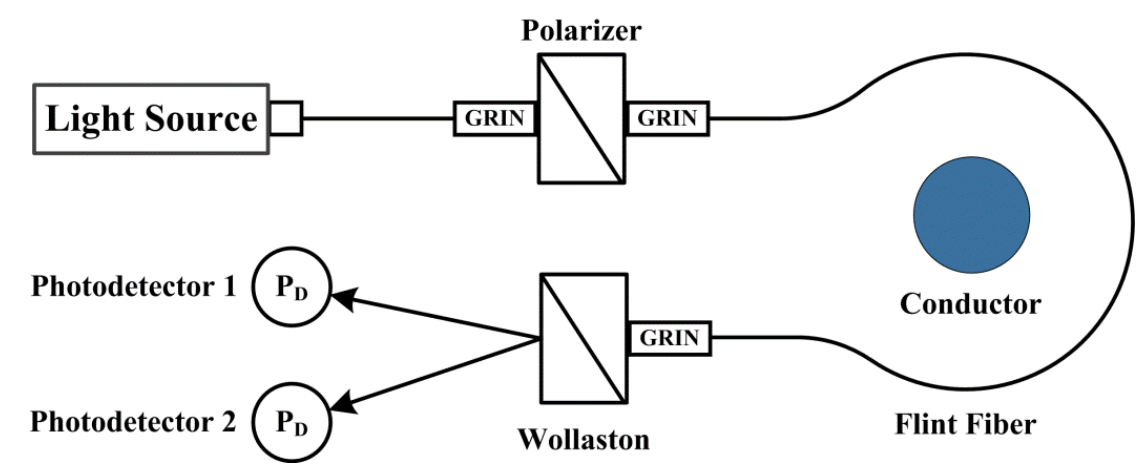

A comparison between the performances of flint glass fiber and twisted single-mode fiber (usually used before the flint glass) as a Faraday element was presented [10], and showed that the system with the flint glass fiber is more stable than the one with twisted fiber.

In 2009, a new optical glass for optical fibers with high refractive index for applications in optical current sensors was reported. The results showed that these fiber glasses could have ten times higher Verdet constant and small internal mechanical stress [11].

Recently, a terbium-doped-core phosphate optical fiber with a Verdet constant six times larger than standard optical fibers was presented [12]. The same authors also presented in 2010 a high concentration of terbium-doped fiber with a record Verdet constant of $-32 \mathrm{rad} /(\mathrm{T} \mathrm{m})$, which is 27 times larger than standard optical fibers and corresponds to $83 \%$ of the Verdet constant of commercially available crystals used in bulk optics-based isolators [13].

Another problem of using the fiber as the transducer is the effect of the linear birefringence that is induced by mechanical stress (when the fiber is bent for example), thermal stress, manufacture imperfections and other effects. The presence of linear birefringence significantly reduces the sensor sensitivity due to the polarization state degeneration. The linear birefringence can be neglected if the circular birefringence is high enough as we can see from Equation (5). The use of a twisted single mode fiber to impose a circular birefringence in the fiber has been demonstrated [14]. A similar approach is to use spun high birefringence fibers [15]. Linear birefringence can also be reduced by fiber annealing process [16,17]. Theoretical models and experimental measurements for the Verdet constant dispersion in annealed fibers have been presented [18].

Some methods for linear birefringence compensation using reflected light propagation have been presented, such as Faraday rotating mirrors [19] and fiber polarization rotators [20] which also doubles the sensitivity for the same fiber length. In Figure 8, a Faraday rotator mirror is shown. This component leads to a polarization shift of $90^{\circ}$ and light that propagates in one axis is coupled to the other axis, and vice-versa. Since the linear birefringence is a reciprocal effect, the phase difference introduced by the linear birefringence will be compensated [19]. 
Figure 8. Faraday rotator mirror.

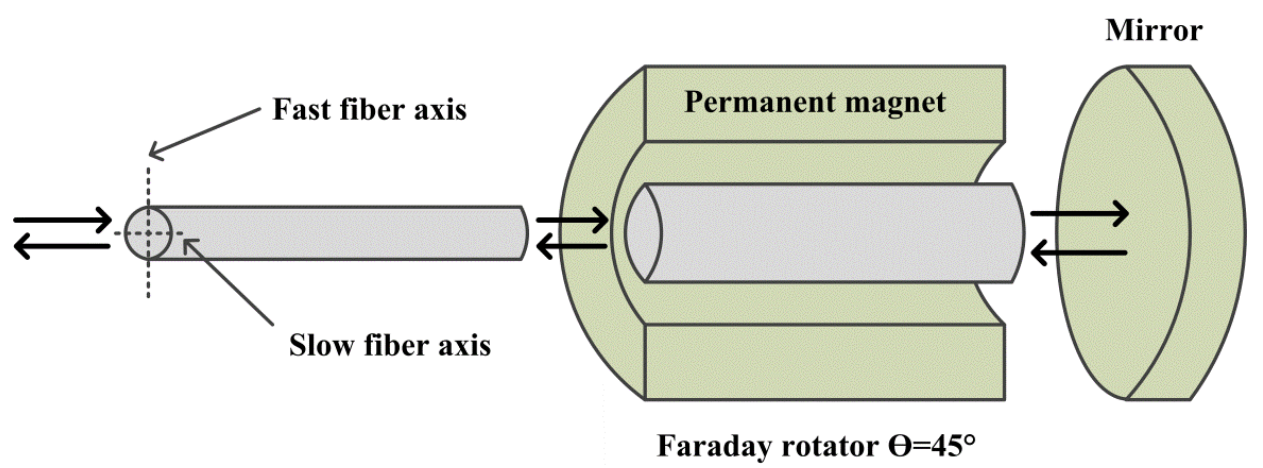

A sensor using reflected light propagation for compensation of linear birefringence, made of lowbirefringent flint fiber with a very low photo-elastic constant, achieved the accuracy required for the $0.1 \%$ class of current metering transformers in the range of $1 \mathrm{kA}[21]$.

Commercial fiber-optic DC current sensors have also been demonstrated [22] being able to measure up to $500 \mathrm{kA}$ with an error of $0.1 \%$. More recently, a signal processing methodology based on artificial neural networks (ANN) was developed, which processes signals from a typical optic current sensors and a thermometer, achieving higher accuracy with temperature and non-linearity compensation [23]. The great benefit of ANN is to get a transfer function for the measurement system taking in account all variables, even those from unwanted and unknown effects, providing a compensated output, after the ANN training session.

Some optical electric current sensors are already commercially available. Considering all-fiber current sensors, for instance, ABB and NXTphase are two companies that have available sensors with working principle identical to the one shown in Figure 6. The first company assures a range of operation up to $500 \mathrm{kA}$ and $0.1 \%$ accuracy [24]. The second company permits DC and AC measurements with a range of operation from 1 to $63 \mathrm{kA}_{\mathrm{RMS}}$ being also in the $0.1 \%$ class category $[25,26]$.

\subsection{Bulk-Optic Current Sensors}

As seen before, all-fiber current sensors are easy to implement and to increase the sensitivity, but suffer from high linear birefringence and have relative low Verdet constants. In this context, bulk optic sensors rapidly imposed and are the most industrial implemented technology $[1,27]$.

When compared to all-fiber devices, bulk optic sensor presents some obvious advantages. These are usually smaller devices and mechanically stiffer. Therefore, mechanical and thermal gradients, vibrations and other external noises in the bulk optic material are very small. Also their Verdet constants are typically, 2 times higher than the ones found in optical fibers, and due to their low photo-elastic coefficients, the intrinsic linear birefringence is also very small, which allows for sensors with high sensitivities. Another big advantage is that the sensor around the conductor does not have to be a single piece of material. This allows an easy installation without the need of interrupting the current in the conductor.

The main disadvantages of these sensors are related to the fact that reflections need to occur inside the bulk optic material for light to go around the conductor. Since the reflection angle will be 
dependent on the refractive index of the crystal and outside surrounding medium (such as air), the sensor will be affected by a number of external factors such as humidity. This can be solved by isolating the sensor from external factors but will add higher cost to installation and maintenance operation of the sensor [28]. Also, every internal reflection of the light that occurs will introduce an optical phase difference between the two polarizations. In a polarimetric or interferometric scheme, this leads to an unavoidable error source [29].

The problem can be solved with the use of double reflections in each corner of the bulk optic material, where the optical phase difference introduced in the first reflection is compensated by the second reflection. A patent describing this idea was registered in 1986 [30] and it is shown in Figure 9.

Figure 9. Bulk optic current sensor with double reflection.

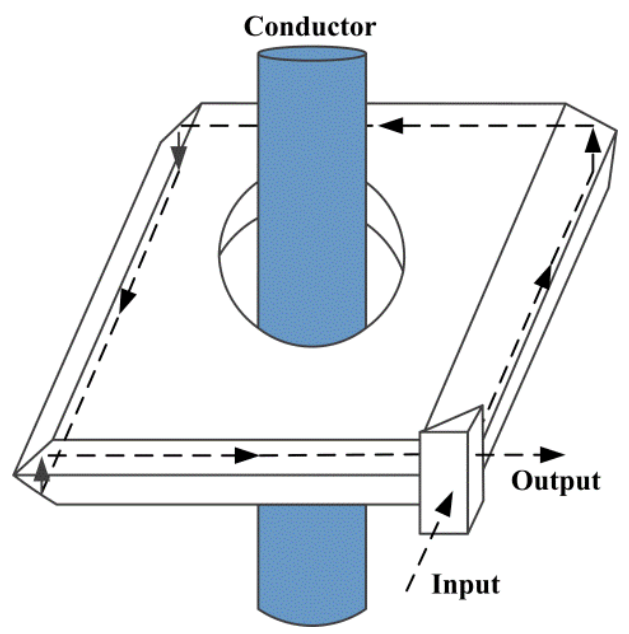

However, this idea presents another problem: in the optical path between the first and second reflections, the polarization state is elliptical and the signal will be non-linearly affected by the magnetic field to be measured. This will cause the system to be affected by external magnetic fields. This problem takes special relevance when measuring current in three phase systems, since the three conductors are usually close to each other. Studies have also been developed to minimize the crosstalk between different conductors [6]. Another possible solution is using a triangular shaped bulk optic material in which the light is always reflected at the critical angle [31], as shown in Figure 10.

Figure 10. Bulk optic triangular shape current sensor.

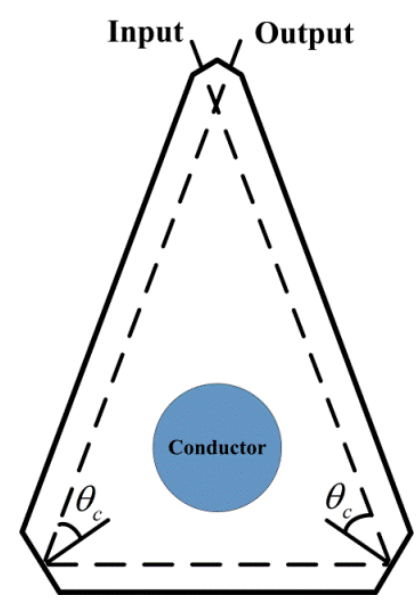


This configuration is simpler than the previous one. However this requires a precise reflection angle cut and a near perfect bulk optic material well isolated from external noise sources. Several schemes have been proposed to further increase the sensitivities of these sensors, including field concentrators (which consist in an air-gap core surrounding the conductor) and extending the light optical path inside the crystal (by using total internal reflections before the light exits from the sensing element) [32]. For this purpose, a circular sensor head was developed, where light is injected through a prism and at specific angles, light travels five times before exiting the prism. This scheme enhances sensibility and it is not sensitive to external fields [33]. Other sensors are available in a square configuration where light passes around the conductor several times [34,35].

Also, in order to increase the sensitivity of the sensor, the compensation of the linear birefringence is very important. It was shown that the use of a Faraday rotator mirror can reduce the angle of rotation of the polarization plane due to the linear birefringence from $20^{\circ}$ to $5^{\circ}[36]$.

The dependence of the Verdet constant with source wavelength is also important, because it changes the sensitivity of the sensor [37]. Also, it was shown that the errors introduced by a small bandwidth optical source are very small and therefore it is reasonable to use the models which assume a monochromatic light source [38]. As in the all-fiber optic sensors, bulk-optic ones also have temperature dependency of the Verdet constant. A solution was measuring the temperature and compensating its effect by introducing a correction factor that depends on the temperature [39].

Many interesting ideas for optical current sensing may be found in literature, but many are not designed for high voltage and/or high current sensing operations. However, specific applications for high current [40] and high voltage [41] can be found. In the case of high currents, the sensors are usually limited to measurements between $0^{\circ}$ and $360^{\circ}$, in order to avoid rotation angle ambiguities. This problem was solved [40] by using a technique which counted the number of times that the phase crossed $0^{\circ}$ and allowed to measure up to $720 \mathrm{kA}$.

Also this type of sensor has been successfully introduced to the market. PowerSense [42] is a company that have a commercial product based on the sensing scheme like the one shown in Figure 2, which permits AC current measurement from 20 to $20,000 \mathrm{~A}$, with maximum errors of $2 \%$.

\subsection{Magnetic Force Sensors}

Optical fiber magnetic field sensors exploit the magnetostrictive effect that produces a mechanical strain in a ferromagnetic material when it is subjected to an external magnetic field. For that an optical fiber is bonded to a sample, or by thin-film deposition with a magnetostrictive material. The most commonly used magnetostrictive sensing elements were optical fibers coated with nickel [43], metallic glasses such as Metglass 2605S2 or Vitrovac 40-60 [44-47] and ceramic thin-films $\left(\mathrm{Fe}_{2} \mathrm{O}_{4}, \mathrm{NiFe}_{2} \mathrm{O}_{4}\right.$ or $\left.\mathrm{Ni}_{\mathrm{x}} \mathrm{Co}_{1-\mathrm{x}} \mathrm{Fe}_{2} \mathrm{O}_{3}\right)[48,49]$.

Since 2000, an intensive investigation on an alloy material with high magnetostriction coefficient (Terfenol-D) has been reported on literature. The most studied optical configuration is a rod or ribbon shape of Terfenol-D attached to a fiber Bragg grating (FBG) [50]. The advantages of this type of sensors are the small size and possibility to have various sensing elements in the same optical fiber, thus allowing optical multiplexing. Alloys using Terfenol-D have also been tested in microstructured 
fibers [51]. Recently another kind of sensors to detect magnetic fields has been proposed, where the sensor is based on magnetic fluid.

\subsubsection{Magnetostrictive Sensors}

During the 80's decade a great effort done for detecting magnetic fields and/or electric current using optical fibers in combination with materials that exhibit magnetostriction effects. In 1980, Yariv et al [43] studied the possibility of detecting weak magnetic fields by using magnetostrictive perturbation in optical fibers. A low loss optical fiber of length $L$ was wrapped around a nickel jacket that suffers a longitudinal mechanical strain when immersed in a magnetic field.

The first experimental evidence of the magnetic field sensing characteristics of optical fibers jacketed with either nickel or metallic glass magnetostrictive materials was reported by Dandrige et al. [44]. Both bulk magnetic stretchers, as well as, thin films directly deposited on a single mode fiber (SMF) were analyzed. An all-fiber Mach-Zehnder interferometer was used to detect the magnetically induced mechanical changes in the optical path length that contained the magnetostrictive jacket. Figure 11 shows the schematic diagram of the magnetometer proposed by Dandrige et al.

Figure 11. Experimental scheme of fiber optic magnetic sensor.

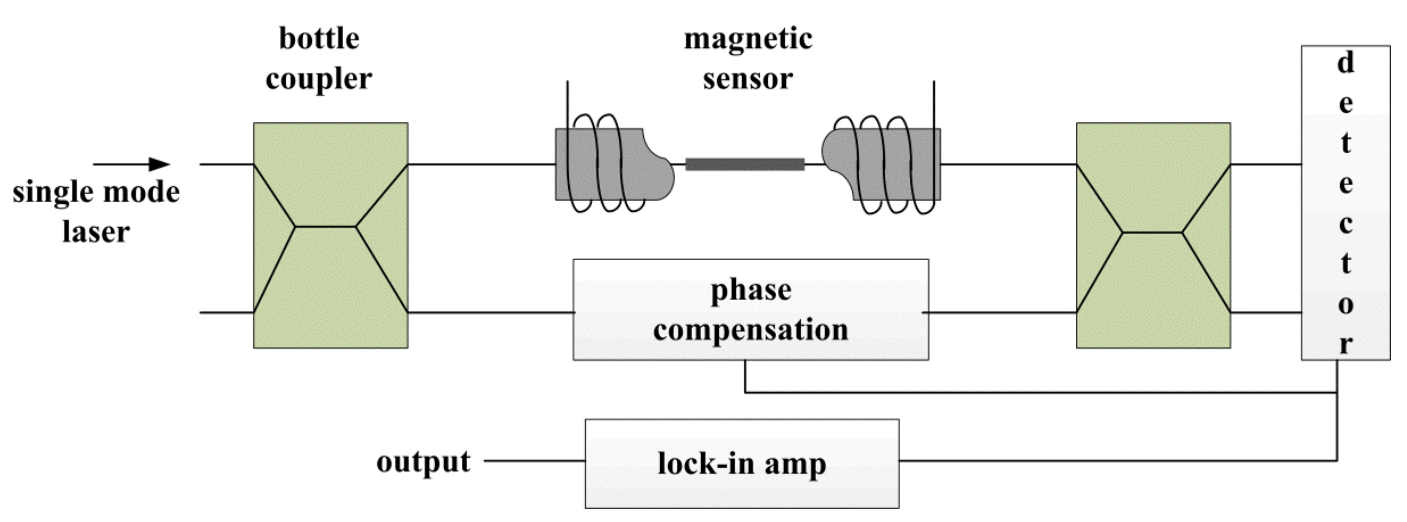

The sensor sensitivity achieved with the bulk nickel material was $6.37 \times 10^{-6} \mathrm{~A} / \mathrm{m}^{2}$. The response of the first generation thin film coated fiber optic sensors was about two orders of magnitude lower and was ruled by the thin-film thickness. Jarzynski et al. [48] investigated the mechanical strain induced by a weak axial magnetic field in an optical fiber with magnetostrictive jacket with different thickness. The sensitivity was calculated as function of jacket thickness for a variety of magnetostrictive materials.

These configurations could be sensitive to magnetic fields, but unfortunately also sensitive to all other parameters that influence the optical path length of the fiber, such as, temperature [52]. Heaton proposed a non-magnetostrictive metal-coated element in one arm of the interferometer to directly detect temperature, improving this way the sensitivity to magnetic field of fiber optic interferometers.

A SMF wounded under tension around a magnetically sensitive nickel cylindrical piece was proposed by Rashleigh [53]. The magnetic field changed the state of polarization (SOP) of light in the fiber. An optical phase sensitivity of $1.76 \times 10^{-2} \mathrm{rad} / \mathrm{m}$ Oe was achieved, allowing a detection of magnetic fields as small as $3.5 \times 10^{-4} \mathrm{~A} / \mathrm{m}^{2}$ of fiber. 
The magneto-optic coupling coefficient as function of frequency for a Mach-Zehnder interferometer incorporating a nickel toroid was measured by Cole et al. [54]. They observed that the frequency response of the coupling coefficient was found to be essentially smooth in a range between 15 and $600 \mathrm{~Hz}$. Hartman et al. [55] reported the fabrication and characterization of optical fibers coated with nickel thin-film. The thickest coating was greater than $90 \mu \mathrm{m}$ and was fabricated by chemical vapor deposition (CVD). The optical phase sensitivity obtained by the fiber Mach-Zehnder interferometer was $1.9 \times 10^{-8} \mathrm{rad} . \mathrm{m} / \mathrm{A}$.

The use of a metallic glass as sensing element for detection of very low magnetic fields was described by Koo and Sigel [45]. In their experiment, different metallic glasses were used on an all fiber Mach-Zehnder interferometer in order to measure their magnetic field detection sensitivities. A minimum detectable magnetic field of $3.98 \times 10^{-7} \mathrm{~A} / \mathrm{m}^{2}$ was reported.

A configuration for measuring DC magnetic fields using an optical fiber coated with amorphous $\mathrm{Fe}_{0.8} \mathrm{~B}_{0.2}$ alloy in one arm of a modified magnetometer was developed by Willson and Jones [56]. In order to obtain a maximum sensitivity, the system was analyzed in quadrature allowing the elimination of polarizations fluctuations. A linear region was analyzed and a sensitivity of $102 \mathrm{rad} / \mathrm{T} \cdot \mathrm{m}$ was obtained.

A technique for the measurement of weak DC and low frequency AC magnetic fields using an all fiber SMF magnetometer was described by Kersey et al. [57]. For the first time, the experimental scheme used, consisted in a Michelson interferometer where the two cleaved fibers ends were coated with silver film to form the mirrors. Detection sensitivities of $\approx 1 \times 10^{-7} \mathrm{~T} / \mathrm{m}$ at $20 \mathrm{~Hz}$ was achieved by the bulk nickel magnetostrictive element. Figure 12 represents the experimental configuration used.

Figure 12. Scheme of the all fiber magnetometer by Kersey et al. [57].

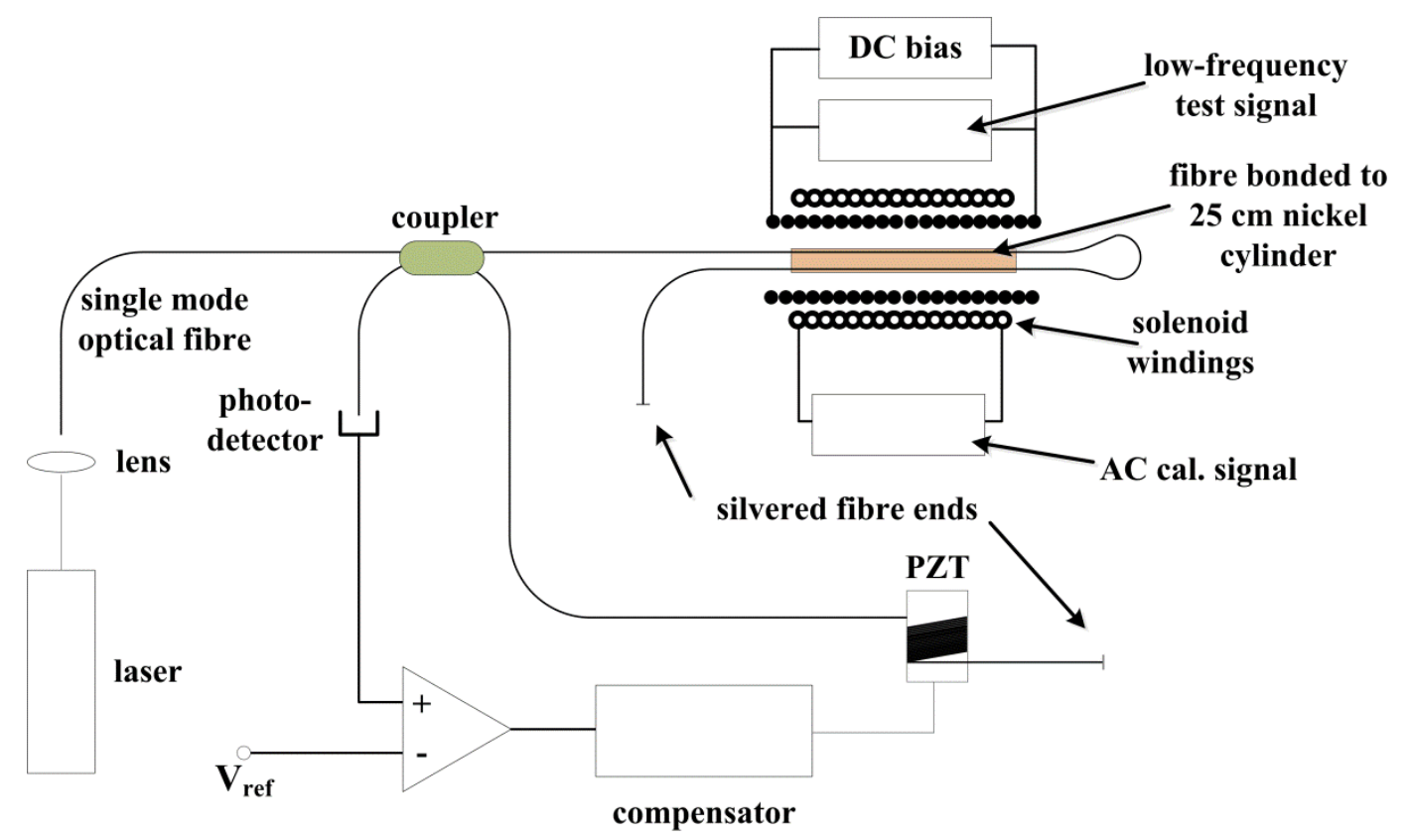

A fiber Mach-Zehnder interferometer passively stabilized using a $3 \times 3$ fiber coupler was used by Koo et al. [58]. The system response was examined by subjecting the metallic glass to a DC and AC magnetic fields. The sensor sensitivity was in the order of $7.96 \times 10^{-5} \mathrm{~A} / \mathrm{m}^{2}$ for a fiber length of $1 \mathrm{~m}$ at $1 \mathrm{~Hz}$ bandwidth. 
The first demonstration of a closed-loop fiber optic magnetometer with dynamic magnetostrictive response was reported by Kersey et al. [59]. The sensor was capable of detecting low frequency and DC changes of magnetic field with a sensitivity of $\approx 2 \mathrm{nT}$ at frequencies below $2 \mathrm{~Hz}$.

One year later, the same group proposed a similar configuration, where the main difference in the magnetometer was that they kept at constant value the total local field (bias+ambient) [46]. By active-bias field stabilization they ensure that the magnetostrictive sensing element was maintained in a zero state zero magnetization. This method eliminated any problems arrived due to magnetic hysteresis of the metallic glass materials used (Metglass and Vitrovac). A minimum detectable of $8 \times 10^{-5} \mathrm{~A} / \mathrm{m}$ was observed over the frequency range from $\mathrm{DC}$ to $20 \mathrm{~Hz}$ using a small length of optical fiber $(\approx 0.5 \mathrm{~m})$.

The effects of external perturbations, such as, mechanical stress and vibrations on the operation of fiber optic magnetometers were investigated by Bucholtz et al. [47]. These perturbations were controlled by maintaining the DC magnetic field at prescribed levels. The analysis performed has shown that the method used by the authors could be applicable in all interferometric fiber sensors employing nonlinear transducing mechanisms.

In 1989, the first demonstration of the detection of magnetic fields at frequencies above $50 \mathrm{kHz}$ using a fiber optic magnetometer was reported by Bucholtz et al. [60]. A Mach-Zehnder interferometer with a thick cylindrical metallic glass transducer in one of the fiber arms was used. Mixing high frequencies signals in the magnetostrictive sensor allowed a heterodyne detection scheme, where the transducer acted as both the receiving and nonlinear mixing element. The same group reported a minimum detectable AC magnetic field of $70 \mathrm{fT} / \sqrt{ } \mathrm{Hz}$ at $34.2 \mathrm{kHz}$ [61].

Optical fibers coated with magnetostrictive ceramic films were tested by Sedlar et al. [49], by using a modified Mach-Zehnder interferometer operating in an open-loop configuration. The sensors exhibited excellent linearity and good sensitivity. The materials used were magnetite, $\mathrm{Fe}_{2} \mathrm{O}_{3}$, nickel ferrite and cobalt doped nickel ferrite $\left(\mathrm{NCF}_{2}\right)$ jackets, being the last one with the best response to magnetic field. They achieved a minimum detectable magnetic field of $3.2 \times 10^{-3} \mathrm{~A} / \mathrm{m}$ for optical fibers jacketed with $2 \mu \mathrm{m}$ thick and $1 \mathrm{~m}$ long $\mathrm{NCF}_{2}$ material.

A fiber optic sensor for measuring DC magnetic fields based on the extrinsic Fabry-Perot interferometer (EFPI) was proposed by $\mathrm{Oh}$ et al. [62]. A SMF and a Metglass wire magnetostrictive transducer constituted the input-and-output and reflector arms of the extrinsic Fabry-Perot interferometer (EFPI) sensor. Due to the sensor optical geometry design, it showed a low vibration sensitivity and high thermal induced compensation (better than 99\%).

Pérez-Millán et al. [63] demonstrated a new approach for measuring electric current on high voltage systems. The fiber optic sensor employed the intrinsic magnetostriction of the ferromagnetic core of a standard current transformer and was interrogated with a Mach-Zehnder interferometer. The sensor avoided certain defects of Faraday based optical current sensors reported, such as, optical power source fluctuations, phase drifts and polarization dependency. Figure 13 represents the experimental configuration used by Pérez-Millán et al. 
Figure 13. Experimental arrangement used for measuring electric current on high voltage systems.

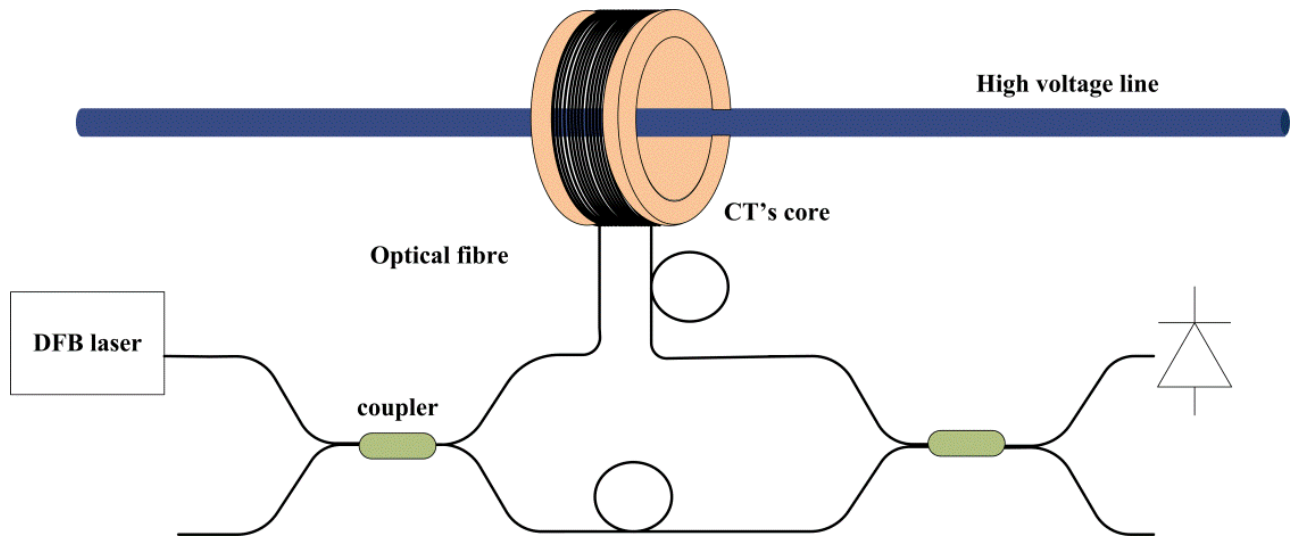

More recently, Djinovic et al [64] presented results of measurement of AC and DC magnetic fields by using a fiber optic interferometric sensor for structural health monitoring. The principle of operation was based on changes in the optical path length of the cavity between a magnetostrictive wire and a fiber optic tip. The sensing configuration consisted of a Michelson fiber interferometer using a $3 \times 3$ single mode fiber coupler. They were able to detect the instant separation between the wire and fiber end with an accuracy of $50 \mathrm{~nm}$ corresponding to a magnetic field in a range of $50 \mathrm{nT}$ to $800 \mu \mathrm{T}$.

\subsubsection{Terfenol-D}

The first work combining Terfenol-D alloy with optical fiber sensors to measure static magnetic fields was reported by Mora et al. [50], see Figure 14. The magnetostrictive sensor with temperature compensation scheme was composed by two different alloys with similar thermal expansion coefficient, being one of them Terfenol-D and the other Monel 400. The mechanical expansion of both materials due to temperature and magnetic field variations was detected by the two FBGs attached. The spectral difference between the two Bragg wavelengths was proportional to the amplitude of the magnetostriction, and, the wavelength shift produced by the grating bonded to the non-magnetic alloy (Monel 400) was proportional to the temperature variation.

Figure 14. Schematic diagram of the fiber sensor proposed by Mora et al. [50].

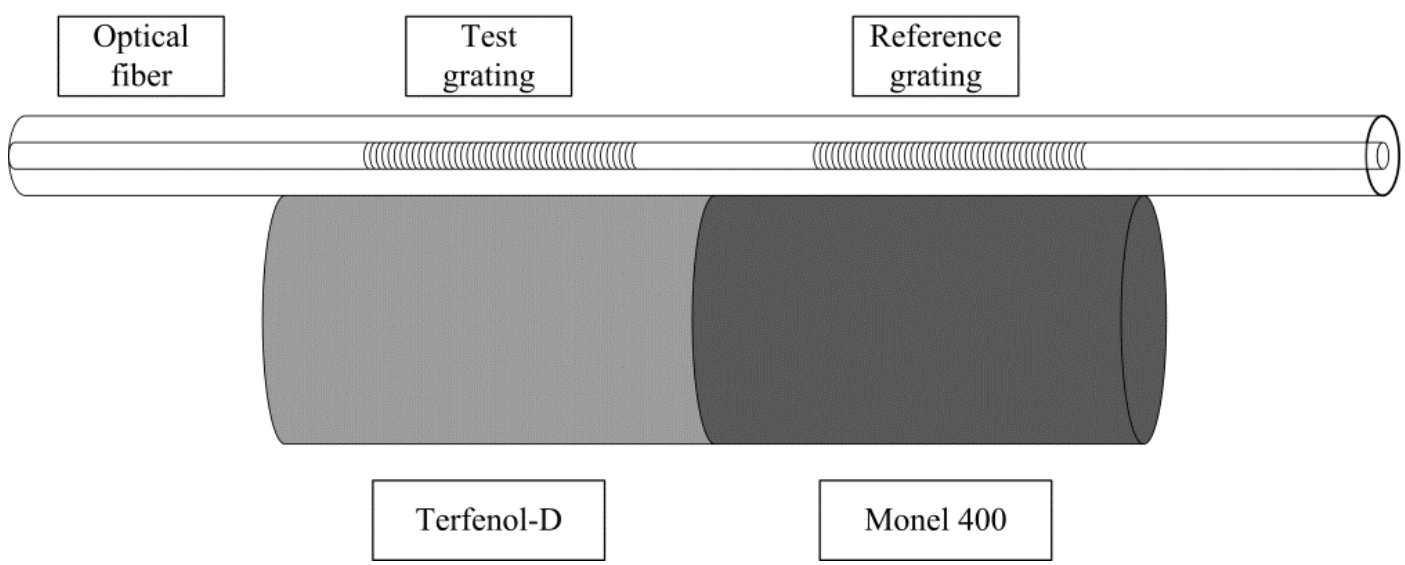


For applied magnetic fields smaller than $4.7 \times 10^{-4} \mathrm{~A} / \mathrm{m}(\mathrm{B}<60 \mathrm{mT})$ a linear response of the fiber sensor was verified. The spectral sensitivity in this range was independent of temperature, having a value of $(2.31 \pm 0.05) \times 10^{-5} \mathrm{~nm} /\left(\mathrm{A}^{2} \mathrm{~m}^{-2}\right)$. In order to compensate the temperature effects in the FBG based on magnetostrictive materials, two simple techniques were demonstrated by Yi et al. [65]. The first technique consisted of two FBGs placed perpendicular to each other and bounded onto a single Terfenol-D layer material. The second technique, two FBGs were stacked onto two different magnetostrictive bars (Terfenol-D and nickel) and physically parallel with each other. The materials used had similar thermal expansion coefficients but with magnetostrictive coefficients of opposite signs. The two techniques were capable of measuring the magnetostrictive effects with temperature insensitivity. The sensitivities due to the magnetic field were $2.44 \times 10^{-4} \mathrm{~nm} / \mathrm{mT}$ and $1.8 \times 10^{-4} \mathrm{~nm} / \mathrm{mT}$, for the first and second techniques, respectively.

An electric current optical sensor based on a passive prototype magnetostriction device for high power applications was developed and demonstrated by Satpathi et al. [66]. The optical current sensor concept was achieved by attaching a piece of Terfenol-D to a FBG, thus producing mechanical strain that was proportional to the magnetic field amplitude. In order to obtain a linear response from the magnetostrictive alloy, the material was subjected to mechanical pre-stress and DC magnetic field bias tuning. The sensor had a linear electrical current range from 100-1,000 A, with a measured phase shift of $30^{\circ}$ for a frequency signal at $60 \mathrm{~Hz}$.

Li et al [67] demonstrated a magnetic field sensor based on dual FBG configuration consisting essentially in a rod of Terfenol-D attached to the optical fiber. One of the gratings was fixed on both ends of the magnetostrictive alloy, while the other was only attached on one point, being the other end free to move. The configuration of dual FBGs was employed for point measurement reference and for easy temperature compensation. The maximum sensitivity achieved was $0.018 \mathrm{~nm} / \mathrm{mT}$ when the applied magnetic flux density was smaller than $70 \mathrm{mT}$.

In 2006, Mora et al. [68] presented a fiber optic AC current sensor for high voltage lines based on an uniform FBG mounted on a Terfenol-D piece. An innovative way of processing the optical signal from the sensing head allowed a simultaneous measurement of temperature and the AC current. The first physical parameter was coded on wavelength shift, while the second one was coded on the amplitude of the signal. The sensor could also operate at long distances with a capacity to be multiplexed.

Also a sensor for simultaneous measurement of $\mathrm{AC}$ current and temperature was reported by Reilly et al. [69]. The device was composed of a magnetically biased Terfenol-D piece fixed to one FBG. The sensor was capable of measuring $\mathrm{AC}$ current at temperatures from $18{ }^{\circ} \mathrm{C}$ to $90{ }^{\circ} \mathrm{C}$. The nonlinear effects originated by the magnetostrictive alloy were identified and methods for compensating these effects were proposed.

A hysteresis compensation technique for a magnetic field sensor, containing a magnetostrictive alloy device, was presented by Davino et al. [70]. The sensing head integrated a rod of Terfenol-D and a FBG. Due to the nonlinear effects taking place in such materials the magneto elastic material was accurately modeled in order to compensate for hysteresis. The algorithm allowed the improvement in the sensor performance.

In 2009 and for the first time, a fiber optic magnetic field sensor with a thin film of Terfenol-D instead of the bulk magnetostrictive materials was reported by Yang et al. [71] as shown in Figure 15. 
By magnetron sputtering deposition process, Terfenol-D thin films were deposited on etched FBGs. Two methods to improve the sensitivity were demonstrated. In one, the magnetostrictive alloy was deposited over different diameters of cladding-etched FBGs. The maximum sensitivity response to magnetic field was $0.95 \mathrm{pm} / \mathrm{mT}$ for an $85 \mu \mathrm{m}$ diameter sensor. The other method was coating FBGs with a layer of magnetostrictive alloys (FeNi and Terfenol-D) and a multilayer with both materials. The multilayer has the highest sensitivity of $1.08 \mathrm{pm} / \mathrm{mT}$. In this experiment, the authors not only reduced the dimensions of the sensor, but also improved the sensitivity to magnetic field.

Figure 15. Configuration of optical fiber magnetic sensor used by Yang et al. [71].

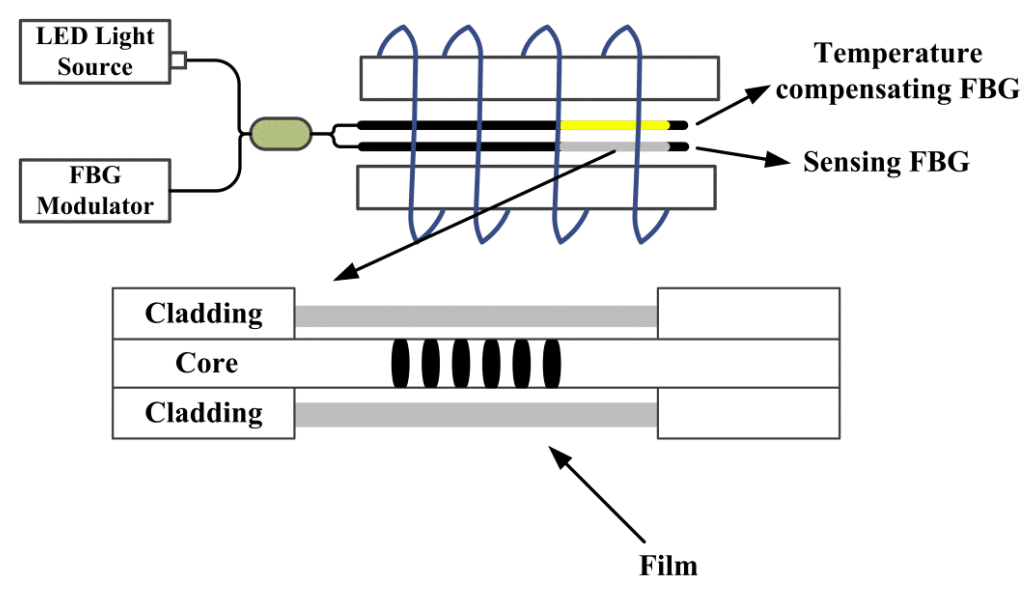

The dependence of shape anisotropy on the strain response of magnetic field sensors based on magnetostrictive alloys and FBGs was studied by Pacheco et al. [72]. The maximum sensor sensitivity was $18 \mu \varepsilon / \mathrm{mT}$ when a biased uniform field was applied for a $20 \mathrm{~mm}$ long Terfenol-D cuboid shape. For gradient magnetic fields, they were able to detect a significant change in the magnetostrictive response at different positions, attaching FBGs along the cuboid containing the magnetic field direction.

Quintero et al. [73] presented a sensor for DC and AC magnetic field measurements. The grating was coated with a layer of a composite mixed with particles of Terfenol-D. Different compositions were tested and the best magnetostrictive response was using a 30\% volume fraction of Terfenol-D mixed in a matrix of epoxy resin as blinder. The effect of a compressive pre-stress in the sensor was also investigated. They achieved a resolution of $0.4 \mathrm{mT}$ without and $0.3 \mathrm{mT}$ with pre-stress. The sensitivity obtained for the magnetic field was $2.2 \times 10^{-6} \mathrm{mT}^{-1}$, using an FBG interrogation system.

Recently, Smith et al. [74] presented a novel sensor for detect static magnetic fields using a inscribed FBG and a micromachined slot sputtered with Terfenol-D. The grating and the slot were created with a femtosecond laser inscribing. The sensitivity to magnetic field in transmission was $0.3 \mathrm{pm} / \mathrm{mT}$. They also measured in reflection with different polarization states achieving sensitivities between $0.2 \mathrm{pm} / \mathrm{mT}$ and $0.1 \mathrm{pm} / \mathrm{mT}$. The use of a femtosecond laser to inscribe a FBG and create a micromachined slot simplifies greatly the fabrication process.

The first fiber optic sensor for measurement of magnetic field by integrating a highly birefringent photonic crystal fiber (HiBi PCF) combined with a Terfenol-D epoxy resin composite material was reported by Quintero et al. [51]. An in fiber modal interferometer was assembled by evenly exciting both eigenmodes of the HiBi PCF. Changes in the cavity length as well as the effective refractive 
index were induced by exposing the sensing head to magnetic fields. The sensitivity obtained was $0.006 \mathrm{~nm} / \mathrm{mT}$ to a range of $300 \mathrm{mT}$. Also a FBG coated with the same composite material was fabricated. For this sensor, the sensitivity achieved was $0.003 \mathrm{~nm} / \mathrm{mT}$.

\subsubsection{Magnetic Fluid}

In 2001, Yang et al. [75] investigated the physical mechanism of the optical transmission of magnetic fluid films under magnetic fields. Originally dispersed, the magnetic particles agglomerated forming magnetic columns in the presence of the magnetic field. The liquid phase was transparent, whereas the columns were opaque. Therefore, the liquid phase dominates the optical transmission of the magnetic fluid film. When the magnetic field strength was raised more columns were formed leading to a decrease in the optical transmission. Later on, the same group investigated the refractive index of the magnetic fluid film and founded to be magnetically modulated in the presence of an external magnetic field [76,77].

In 2007 Liu et al. [78] reported a magneto optic tunable filter based on a long period fiber grating (LPG) coated with magnetic fluid as environment media. Applying a variable magnetic field, the center wavelength shift of the attenuation band of LPG was about $7.4 \mathrm{~nm}$. The refractive index dependence of magnetic fluid on the external magnetic intensity was measured and the simulation results exhibited a good agreement.

A fiber optic current sensor based on the magnetic fluid was developed by $\mathrm{Hu}$ et al. [79]. The magnetic fluid was used as the medium in a Fabry-Perot (FP) resonant cavity. The refractive index characteristic of the magnetic fluid suffers variations due to the external magnetic field and the current was measured by the output wavelength of the FP fiber sensor. A signal demodulation method using a FBG wavelength measurement system was proposed. The results indicated a good linearity and, the thickness and initial concentration of the magnetic fluid affected the performance of the sensor.

Dai et al. [80] investigated a novel fiber optic sensor based on magnetic fluid and etched gratings. The nanoparticles of $\mathrm{Fe}_{3} \mathrm{O}_{4}$ (the magnetic fluid used) were injected into capillaries containing etched FBGs acting as sensing elements. The grating with smaller diameter exhibited a wavelength shift of $86 \mathrm{pm}$, being the most sensitive. Experimental results showed a reversible response to magnetic field under $16 \mathrm{mT}$.

A magnetic field sensor consisting of a photonic crystal fiber and small amount of $\mathrm{Fe}_{3} \mathrm{O}_{4}$ magnetic nano-fluid trapped in the cladding holes of a polarization maintaining photonic crystal fiber were reported by Thakur et al. [81]. They demonstrated that magnetic field of few $\mathrm{mT}$ can be easily and very well detected with higher sensitivity of $242 \mathrm{pm} / \mathrm{mT}$.

Later on, a magneto optic modulator with a magnetic fluid film inserted on a Sagnac fiber interferometer was proposed by $\mathrm{Zu}$ et al. [82]. When the magnetic field was applied, the magnetic fluid exhibited a variable birefringence, and this Faraday Effect led to phase and polarization state variations.

Zhao et al. [83] reported the use of hollow core photonic crystal fiber (HC PCF) in the fabrication of the cavity of the FP sensor, as shown in Figure 16, which exhibits low transmission losses and insensitivity to temperature variations. The core of the HC PCF was filled with magnetic fluid allowing this way its use as a magnetic sensor. The sensitivity achieved was $0.41 \mathrm{pm} /(\mathrm{A} / \mathrm{m})$. 
Figure 16. Structure diagram of magnetic filled HC PCF FP sensor proposed by Zhao et al. [83].

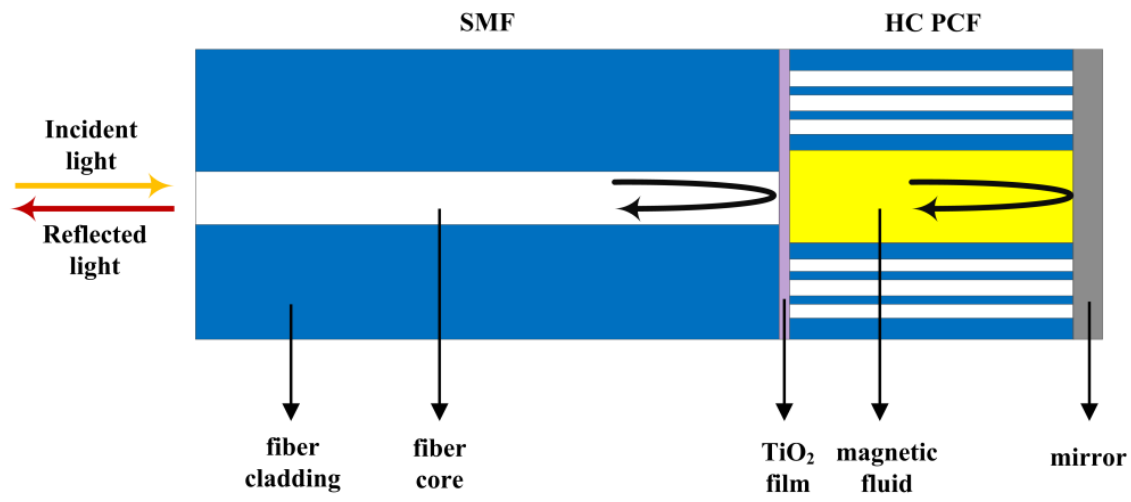

$\mathrm{Zu}$ et al. [84] proposed a new magnetic field fiber sensor design based on magnetic fluids. The sensor was configured as a Sagnac interferometer with a magnetic fluid film and a section of polarization maintaining fiber inserted in a fiber loop. The wavelength shift sensitivity to magnetic field was $0.21 \mathrm{pm} /(\mathrm{A} / \mathrm{m})$ with a resolution of $47.7 \mathrm{~A} / \mathrm{m}$. The optical power also varied with the magnetic field strength obtaining a sensitivity of $5.02 \times 10^{-3} \mathrm{~dB} /(\mathrm{A} / \mathrm{m})$.

\subsection{Hybrid Sensors}

This type of device tries to explore the combination of the optical fiber sensors and the conventional current transducers. The main advantage is that as the insulation is provided by the fiber optic itself, and the cost of the insulation needed for a conventional current transducer would be greatly reduced [1]. Sensing systems using hybrid devices consist mainly of a conventional electrical or electronic current sensor, a current transformer (such as Rogowski coil) or a photo-electronic device, but interrogated by an optical fiber system. The principal element of this combination is an electro-optic converter which transforms electrical current modulation into optical modulation [1].

To control the sensing configurations, diverse optical modulation schemes have been reported in recent years, such as optical phase modulation [85], intensity modulation [86], frequency modulation [87], polarization modulation [1] and chromatic modulation [88]. Figure 17 shows an experimental configuration for this type of sensor.

Figure 17. Typical setup of a hybrid sensor configuration.

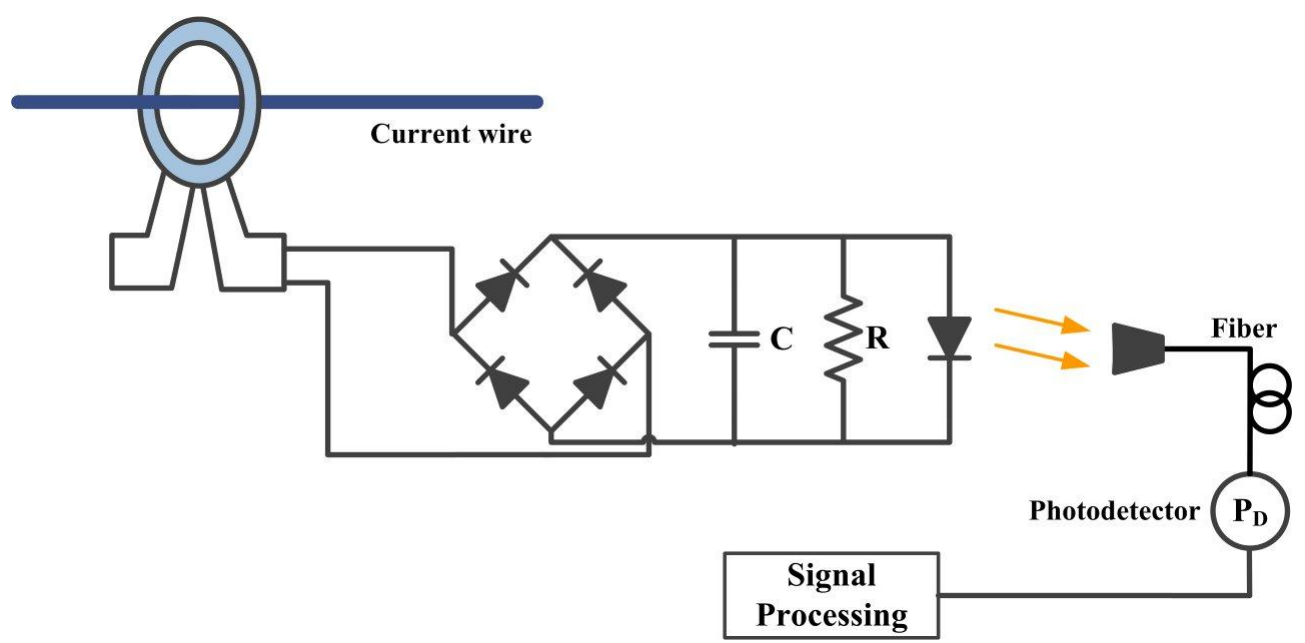




\section{Commercially Available Prototypes}

There are several companies that have commercial products available for high voltage electrical current measuring based in optical systems. The company AIRAK developed an optical fiber based current sensor (Figure 18(a)) for monitoring medium voltage electrical distribution lines. The sensor is very lightweight (about $1.25 \mathrm{lbs}(0.57 \mathrm{~kg}$ )) and small size (about 11 " $\times 7$ " $\times 6.25$ " $(28 \mathrm{~cm} \times 18 \mathrm{~cm} \times 16 \mathrm{~cm}))$. As for the technical details, the sensor meets the IEEE $36 \mathrm{kV}$ insulation class, has a resolution better than $0.5 \%$ f.s., an operating frequency range of $5 \mathrm{~Hz}-5 \mathrm{kHz}$, temperature range of $40{ }^{\circ} \mathrm{C}$ to $+80{ }^{\circ} \mathrm{C}$, and has a full scale measurement range of $1 \mathrm{kA}, 3 \mathrm{kA}$ or $15 \mathrm{kA}$. The DISCOS outdoor current sensor from PowerSense, developed for AC measurement in lines of up to $36 \mathrm{kV}$, has a dynamic range from $5 \mathrm{~A}$ to $20 \mathrm{kA}$ with a measuring accuracy of $2 \%$.

For higher voltage systems, companies like NxtPhase (now Alstom Grid) and ABB present similar solutions to measure electrical current on electrical potentials up to $800 \mathrm{kV}$.

The sensor NXCT from NxtPhase (Figure 18(b)), has a dynamic range from 1 A to 4 kA with performances that exceeds the IEC Class $0.2 \mathrm{~S}$ and IEEE Class 0.3 accuracy $(0.15 \%)$ for metering applications. The sensor is also able to measure up to $63 \mathrm{kArms}$ for short-time current (about $1 \mathrm{~s}$ ) and accurate waveform reproduction up to $6 \mathrm{kHz}$. Different sensor weights, between $108 \mathrm{lbs}$ (49 $\mathrm{kg}$ ) for $72.5 \mathrm{kV}$ and $210 \mathrm{lbs}(95 \mathrm{~kg})$ for $800 \mathrm{kV}$ are supplied. The sensor can operate in a temperature range between $40{ }^{\circ} \mathrm{C}$ to $+55^{\circ} \mathrm{C}$.

As for the sensor MOCT from ABB (Figure 18(c)), it also has a dynamic range from 1 A to $4 \mathrm{kA}$ with accuracy performances that exceed IEC Class 0.2S and ANSI Class $0.15 \mathrm{~s}$ for metering applications. The system provides accurate waveform reproduction up to $100 \mathrm{kA}$. Different sensor weights, between $110 \mathrm{lbs}(50 \mathrm{~kg}$ ) for $72.5 \mathrm{kV}$ and $410 \mathrm{lbs}(186 \mathrm{~kg}$ ) for $800 \mathrm{kV}$ are also supplied.

An optical current sensor from SIEMENS is also presented in Figure 18(d), although technical data are not available.

Figure 18. Commercially available prototypes for high voltage electrical current measuring based in optical systems: (a) AIRAK's Overhead Mountable Optical Current Sensor (b) NxtPhase's NXCT Optical current sensor (c) ABB's MOCT (Magneto-Optical Current Transformer) (d) SIEMENS's Optical current sensor.
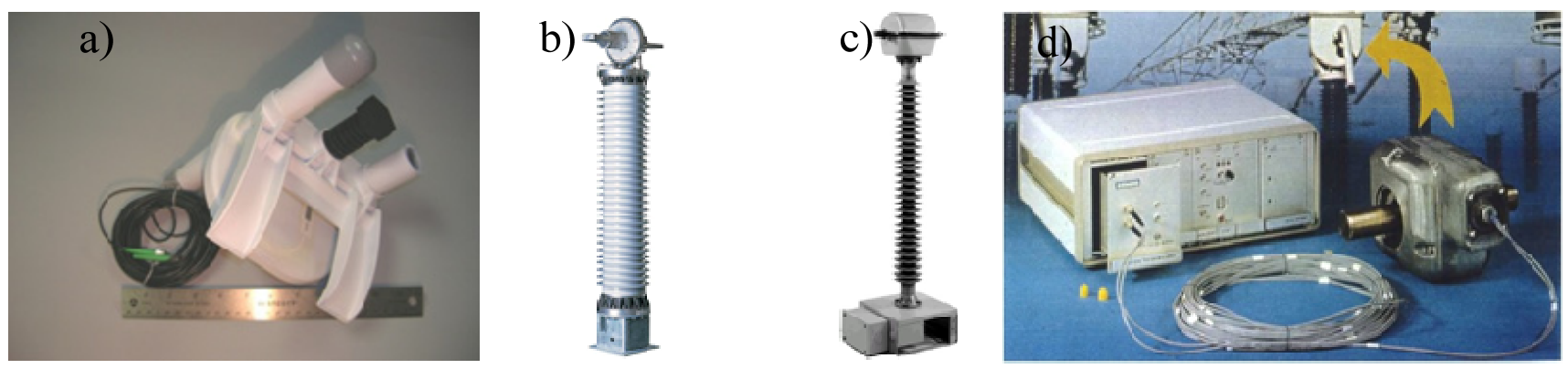

\section{Conclusions}

A review of optical current sensors for high voltage current sensing applications, including the different configurations, physical principles, advantages and disadvantages and state-of-the-art including commercially available systems, was presented. 
In all-fiber sensors, immunity against external currents/magnetic fields can be easily obtained, although linear birefringence can present a serious limitation to the performance of the sensor. Commercially available sensors for DC current measurement have been shown to measure up to $500 \mathrm{kA}$ with $0.1 \%$ accuracy and for AC current measurement up to $63 \mathrm{kA}_{\mathrm{RMS}}$ with $0.1 \%$ accuracy.

Bulk magneto-optical sensors are more robust when compared to all-fiber sensors, being more protected against mechanical and thermal gradients, vibrations and other noises, although immunity against external currents/magnetic fields is harder to obtain. They have also higher Verdet constants and low linear birefringence which allows for high sensitivities over high measurement ranges. Commercially available sensors which allow for AC current measuring from 20 A to 20,000 A with maximum errors of $2 \%$ and sensors which allowed to measure up to $720 \mathrm{kA}$ have been demonstrated.

With regard to magnetic force optical current sensors, the use of magnetostrictive materials, such as Terfenol-D, attached to an FBG in an optical fiber can present an interesting solution. Using this idea, a prototype was able to measure current in a range of 100-1000 A for a frequency signal at $60 \mathrm{~Hz}$. Sensors able to measure DC current have also been demonstrated.

Lastly, Hybrid sensors can be explored in order to try to combine the best properties of conventional technology and new (optical) technology. The insulation provided by an optical fiber could reduce the cost of the insulation needed for a conventional current transducer. A very important issue regarding Hybrid sensors is that they can be used as a first step to start implementing optical current sensors in the industry, before exclusively optical current sensors can be further developed.

\section{References}

1. Ning, Y.N.; Wang, Z.P. Recent progress in optical current sensing techniques. Rev. Sci. Instrum. 1995, 66, 3097-3111.

2. Hecht, E. Optics, 4th ed.; Fundação Calouste Gulbenkian: San Francisco, CA, USA, 2002; pp. 325-368.

3. Jorge, P. Sensores Ópticos para a Medição de Corrente Elétrica em Alta-tensão. Master Thesis; Faculdade de Ciências da Universidade do Porto, Porto, Portugal, 2001.

4. Blake, J.; Tantaswadi, P.; Carvalho, R. In-line sagnac interferometer current sensor. IEEE Trans. Power Delivery 1996, 11, 116-121.

5. Jackson, D.A. An optical system with potential for remote health monitoring of subsea machinery. Meas. Sci. Technol. 2009, 20, 1-8.

6. Perciante, C.D.; Ferrari, J.A. Magnetic crosstalk minimization in optical current sensors. IEEE Trans. Instrum. Meas. 2008, 57, 2304-2308.

7. Kurosawa, K.; Sakamoto, K.; Yoshida, S. Polarization-Maintaining Properties of the Flint GlassFiber for the Faraday Sensor Element. In the Tenth International Conference on Optical Fiber Sensors, Glasgow, Scotland, 11 October 1994; pp. 28-35.

8. Yamashita, T.; Watabe, A.; Masuda, I.; Sakamoto, K.; Kurosawa, K.; Yoshida, S. Extremely Small Stress-Optic Coefficient Glass Single Mode Fibers for Current Sensor. In Optical Fiber Sensors 11, Japan, 21 May 1996; pp. 168-171.

9. Kurosawa, K. Optical current transducers using flint glass fiber as the Faraday sensor element. Opt. Rev. 1997, 4, 38-44. 
10. Hotate, K.; Thai, B.T.; Saida, T. Comparison between Flint Glass Fiber and Twisted/Bent SingleMode Fiber as a Faraday Element in an Interferometric Fiber Optic Current Sensor. In European Workshop on Optical Fibre Sensors, Scotland, 8 July 1998; pp. 233-237.

11. Barczak, K.; Pustelny, T.; Dorosz, D.; Dorosz, J. New optical glasses with high refractive indices for applications in optical current sensors. Acta Phys. Pol. A 2009, 116, 247-249.

12. Sun, L.; Jiang, S.; Zuegel, J.D.; Marciante, J.R. Effective verdet constant in terbium-doped-core. Opt. Lett. 2009, 34, 1699-1701.

13. Sun, L.; Jiang, S.; Marciante, J.R. Compact all-fiber optical Faraday components using 65-wt\%terbium-doped fiber with a record Verdet constant of $-32 \mathrm{rad} /(\mathrm{Tm})$. Opt. Express 2010, 18, 12191-12196.

14. Rose, A.H.; Ren, Z.B.; Day, G.W. Twisting and annealing optical fiber for current sensors. J. Lightwave Technol. 1996, 14, 2492-2498.

15. Laming, R.I.; Payne, D.N. Electric-current sensors employing spun highly birefringent optical fibers. J. Lightwave Technol. 1989, 7, 2084-2094.

16. Bohnert, K.; Gabus, P.; Brandle, H. Towards commercial use of optical fiber current sensors. In Conference on Lasers and Electro-Optics (CLEO 2000), San Francisco, CA, USA, 7-12 May 2000; pp. 303-304.

17. Tang, D.; Rose, A.H.; Day, G.W.; Etzel, S.M. Annealing of linear birefringence in single-mode fiber coils-Application to optical fiber current sensors. J. Lightwave Technol. 1991, 9, 1031-1037.

18. Rose, A.H.; Etzel, S.M.; Wang, C.M. Verdet constant dispersion in annealed optical fiber current sensors. J. Lightwave Technol. 1997, 15, 803-807.

19. Drexler, P.; Fiala, P. Utilization of Faraday mirror in fiber optic current sensors. Radioengineering 2008, 17, 101-107.

20. Zhou, S.; Zhang, X. Simulation of linear birefringence reduction in fiberoptical current sensor. IEEE Photon. Technol. Lett. 2007, 19, 1568-1570.

21. Kurosawa, K.; Yamashita, K.; Sowa, T.; Yamada, Y. Flexible fiber faraday effect current sensor using flint glass fiber and reflection scheme. IEICE Trans. Electron. 2000, 83, 326-330.

22. Bohnert, K.; Philippe, G.; Hubert, B.; Guggenbach, P. Highly accurate fiber-optic DC current sensor for the electrowinning industry. IEEE Trans. Ind. Appl. Mag. 2005, 43, 180-187.

23. Zimmermann, A.C.; Besen, M.; Encinas, L.S.; Nicolodi, R. Improving Optical Fiber Current Sensor Accuracy using Artificial Neural Networks to Compensate Temperature and Minor Non-Ideal Effects. In the 21st International Conference on Optical Fiber Sensors, Ottawa, Canada, 21 May 2011, pp. 77535Q:1-77535Q:4.

24. The ABB Group-Automation and Power Technologies. Available online: http://www.abb.com/ (accessed on 14 December 2011).

25. Rahmatian, F.; Blake, J.N. Applications of high-voltage fiber optic current sensors. In IEEE Power Engineering Society General Meeting, Montreal, Canada, 18 June 2006; pp. 1-6.

26. Alstom Grid. Available online: http://www.nxtphase.com/ (accessed on 12 Dezember 2011).

27. Ripka, P. Electric current sensors: A review. Meas. Sci. Technol. 2010, 21, 1-23.

28. Walsey, G.A.; Fisher, N.E. Control of the Critical Angle of Reflection in an Optical Current Sensor. In Optical Fiber Sensors 12, Williamburg, VA, USA, 28 October 1997; pp. 237-240. 
29. Bush, S.P.; Jackson, D.A. Numerical investigations of the effects of birefringence and total internal reflection on Faraday effect current sensors. Appl. Opt. 1992, 31, 5366-5374.

30. Sato, T.; Takahashi, G.T.; Inui, Y. Method and apparatus for optically measuring a current. Patent Number: 4564754, 1986.

31. Fisher, N.E.; Jackson, D.A. Vibration immunity and Ampere's circuital law for a near perfect triangular Faraday current sensor. Meas. Sci. Technol. 1996, 7, 1099-1102.

32. Yi, B.; Chu, B.; Chiang, K.S. Magneto-optical electric-current sensor with enhanced sensitivity. Meas. Sci. Technol. 2002, 13, N61-N63.

33. Ning, Y.N.; Chu, B.; Jackson, D.A. Miniature Faraday current sensor based on multiple critical angle reflections in a bulk-optic ring. Opt. Lett. 1991, 16, 1996-1998.

34. Ning, Y.N.; Wang, Z.P.; Palmer, A.W.; Gratan, K. A Faraday current sensor using a novel multi-optical-loop sensing element. Meas. Sci. Technol. 1995, 6, 1339-1342.

35. Benshun, Y.; Andrew, C.; Madden, I.; MacDonald, J.R.; Andonovic, I. A Novel bulk-glass optical current transducer having an adjustable multiring closed-optical-path. IEEE Trans. Instru. Meas. 1998, 47, 240-243.

36. Wang, Z.P.; Wang, H.; Jiang, H.; Liu, X. A magnetic field sensor based on orthoconjugate reflection used for current sensing. Opt. Laser Technol. 2007, 39, 1231-1233.

37. Wang, Z.P.; Qing, B.; Q. Yi, J.Z.; Jin, H. Wavelength dependence of the sensitivity of a bulk-glass optical current transformer. Opt. Laser Technol. 2006, 38, 87-93.

38. Wang, Z.P.; Xiaozhong, W.; Liu, X.; Chunmei, O.; Tan, Q. Effect of the spectral width of optical sources upon the output of an optical current sensor. Meas. Sci. Technol. 2005, 16, 1588-1592.

39. Madden, W.I.; Michie, W.C.; Cruden, A.; Niewczas, P.; McDonald, J.R. Temperature compensation for optical current sensors. Opt. Eng. 1999, 38, 1699-1707.

40. Deng, X.Y.; Li, Z.; Qixian, P.; Liu, J.; Tian, J. Research on the magneto-optic current sensor for highcurrent pulses. Rev. Sci. Instru. 2008, 79, 1-4.

41. Cruden, A.; Michie, C.; Madden, I.; Niewczas, P.; McDonald, J.R.; Andonovic, I. Optical current measurement system for high-voltage applications. Measurement 1998, 24, 97-102.

42. PowerSense A/S-DISCOS System. Available online: http://www.powersense.dk/ (accessed on 15 December 2011),

43. Yariv, A.; Winsor, H.V. Proposal for detection of magnetic-fields through magnetostrictive perturbation of optical fibers. Opt. Lett. 1980, 5, 87-89.

44. Dandridge, A.; Tveten, A.B.; Sigel, G.H.; West, E.J.; Giallorenzi, T.G. Optical fiber magnetic-field sensors. Electron. Lett. 1980, 16, 408-409.

45. Koo, K.P.; Sigel, G.H. Characteristics of fiberoptic magnetic-field sensors employing metallic glasses. Opt. Lett. 1982, 7, 334-336.

46. Kersey, A.D.; Jackson, D.A.; Corke, M. Single-mode fibre-optic magnetometer with DC bias field stabilization. J. Lightwave Technol. 1985, 3, 836-840.

47. Bucholtz, F.; Koo, K.P.; Dandridge, A. Effect of external perturbations on fiber-optic magnetic sensors. J. Lightwave Technol. 1988, 6, 507-512.

48. Jarzynski, J.; Cole, J.H.; Bucaro, J.A.; Davis, C.M. Magnetic-field sensitivity of an optical fiber with magnetostrictive jacket. Appl. Opt. 1980, 19, 3746-3748. 
49. Sedlar, M.; Paulicka, I.; Sayer, M. Optical fiber magnetic field sensors with ceramic magnetostrictive jackets. Appl. Opt. 1996, 35, 5340-5344.

50. Mora, J.; Diez, A.; Cruz, J.L.; Andres, M.V. A magnetostrictive sensor interrogated by fiber gratings for DC-Current and temperature discrimination. IEEE Photon. Technol. Lett. 2000, 12, $1680-1682$.

51. Quintero, S.M.M.; Martelli, C.; Braga, A.M.B.; Valente, L.C.G.; Kato, C.C. Magnetic field measurements based on terfenol coated photonic crystal fibers. Sensors 2011, 11, 11103-11111.

52. Heaton, H.I. Thermal straining in a magnetostrictive optical fiber interferometer. Appl. Opt. 1980, 19, 3719-3720.

53. Rashleigh, S.C. Magnetic-field sensing with a single-mode fiber. Opt. Lett. 1981, 6, 19-21.

54. Cole, J.H.; Lagakos, N.; Jarzynski, J.; Bucaro, J.A. Magneto-optic coupling coefficient for fiber interferometric sensors. Opt. Lett. 1981, 6, 216-218.

55. Hartman, N.; Vahey, D.; Kidd, R.; Browning, M. Fabrication and testing of a nickel-coated single-mode fiber magnetometer. Electron. Lett. 1982, 18, 224-226.

56. Willson, J.P.; Jones, R.E. Magnetostrictive fiber-optic sensor system for detecting DC magnetic-fields. Opt. Lett. 1983, 8, 333-335.

57. Kersey, A.D.; Corke, M.; Jackson, D.A.; Jones, J.D.C. Detection of DC and low-frequency AC magnetic-fields using an all single-mode fiber magnetometer. Electron. Lett. 1983, 19, 469-471.

58. Koo, K.; Dandridge, A.; Tveten, A.; Sigel, G., Jr. A fiber-optic DC magnetometer. J. Lightwave Technol. 1983, 1, 524-525.

59. Kersey, A.D.; Corke, M.; Jackson, D.A. Phase-shift nulling dc-field fibre-optic magnetometer. Electron. Lett. 1984, 20, 573-574.

60. Bucholtz, F.; Dagenais, D.M.; Koo, K.P. Mixing and detection of Rf signals in fibre-optic magnetostrictive sensor. Electron. Lett. 1989, 25, 1285-1286.

61. Bucholtz, F.; Dagenais, D.M.; Koo, K.P. High-frequency fiberoptic magnetometer with $70 \mathrm{Ft} /$ square-root (hz) resolution. Electron. Lett. 1989, 25, 1719-1721.

62. Oh, K.D.; Ranade, J.; Arya, V.; Wang, A.; Claus, R.O. Optical fiber Fabry-Perot interferometric sensor for magnetic field measurement. IEEE Photon. Technol. Lett. 1997, 9, 797-799.

63. Perez-Millan, P.; Martinez-Leon, L.; Diez, A.; Cruz, J.L.; Andres, M.V. A fiber-optic current sensor with frequency-codified output for high-voltage systems. IEEE Photon. Technol. Lett. 2002, 14, 1339-1341.

64. Djinovic, Z.; Tomic, M.; Gamauf, C. Fiber-optic interferometric sensor of magnetic field for structural health monitoring. In Eurosensors XXIV Conference, Linz, Austria, 5-8 September 2010; Volume 5, pp. 1103-1106.

65. Yi, B.; Chu, B.C.B.; Chiang, K.S. Temperature compensation for a fiber-Bragg-grating-based magnetostrictive sensor. Microwave Opt. Technol. Lett. 2003, 36, 211-213.

66. Satpathi, D.; Moore, J.A.; Ennis, M.G. Design of a Terfenol-D based fiber-optic current transducer. IEEE Sens. J. 2005, 5, 1057-1065.

67. Li, M.F.; Zhou, J.F.; Xiang, Z.Q.; Lv, F.Z. Giant magnetostrictive magnetic fields sensor based on dual fiber Bragg gratings. In 2005 IEEE Networking, Sensing and Control Proceedings, Arizona, AZ, USA, 19-22 March 2005; pp. 490-495. 
68. Mora, J.; Martinez-Leon, L.; Diez, A.; Cruz, J.L.; Andres, M.V. Simultaneous temperature and ac-current measurements for high voltagelines using fiber Bragg gratings. Sens. Actuat. A Phys. 2006, 125, 313-316.

69. Reilly, D.; Willshire, A.J.; Fusiek, G.; Niewczas, P.; McDonald, J.R. A fiber-Bragg-grating-based sensor for simultaneous AC current and temperature measurement. IEEE Sens. J. 2006, 6, $1539-1542$.

70. Davino, D.; Visone, C.; Ambrosino, C.; Campopiano, S.; Cusano, A.; Cutolo, A. Compensation of hysteresis in magnetic field sensors employing fiber Bragg grating and magneto-elastic materials. Sens. Actuat. A Phys. 2008, 147, 127-136.

71. Yang, M.H.; Dai, J.X.; Zhou, C.M.; Jiang, D.S. Optical fiber magnetic field sensors with TbDyFe magnetostrictive thin films as sensing materials. Opt. Express 2009, 17, 20777-20782.

72. Pacheco, C.J.; Bruno, A.C. The effect of shape anisotropy in giant magnetostrictive fiber Bragg grating sensors. Meas. Sci. Technol. 2010, 21, 065205-065209.

73. Quintero, S.M.M.; Braga, A.M.B.; Weber, H.I.; Bruno, A.C.; Araujo, J.F.D.F. A Magnetostrictive composite-fiber Bragg grating sensor. Sensors 2010, 10, 8119-8128.

74. Smith, G.N.; Allsop, T.; Kalli, K.; Koutsides, C.; Neal, R.; Sugden, K.; Culverhouse, P.; Bennion, I. Characterisation and performance of a Terfenol-D coated femtosecond laser inscribed optical fibre Bragg sensor with a laser ablated microslot for the detection of static magnetic fields. Opt. Express 2011, 19, 363-370.

75. Yang, S.Y.; Chiu, Y.P.; Jeang, B.Y.; Horng, H.E.; Hong, C.Y.; Yang, H.C. Origin of field-dependent optical transmission of magnetic fluid films. Appl. Phys. Lett. 2001, 79, 2372-2374.

76. Yang, S.Y.; Chen, Y.F.; Horng, H.E.; Hong, H.E.; Hong, C.Y.; Tse, W.S.; Yang, H.C. Magnetically-modulated refractive index of magnetic fluid films. Appl. Phys. Lett. 2002, 81, 4931-4933.

77. Yang, S.Y.; Chieh, J.J.; Horng, H.E.; Hong, C.Y.; Yang, H.C. Origin and applications of magnetically tunable refractive index of magnetic fluid films. Appl. Phys. Lett. 2004, 84, 5204-5206.

78. Liu, T.; Chen, X.; Di, Z.; Zhang, J.; Li, X.; Chen, J. Tunable magneto-optical wavelength filter of long-period fiber grating with magnetic fluids. Appl. Phys. Lett. 2007, 91, 121116:1-121116:3.

79. Hu, T.; Zhao, Y.; Li, X.; Chen, J.J.; Lu, Z.W. Novel optical fiber current sensor based on magnetic fluid. Chin. Opt. Lett. 2010, 8, 392-394.

80. Dai, J.X.; Yang, M.H.; Li, X.B.; Liu, H.L.; Tong, X.L. Magnetic field sensor based on magnetic fluid clad etched fiber Bragg grating. Opt. Fiber Technol. 2011, 17, 210-213.

81. Thakur, H.V.; Nalawade, S.M.; Gupta, S.; Kitture, R.; Kale, S.N. Photonic crystal fiber injected with $\mathrm{Fe}_{3} \mathrm{O}_{4}$ nanofluid for magnetic field detection. Appl. Phys. Lett. 2011, 99, 161101:1-161101:3.

82. Zu, P.; Chan, C.C.; Siang, L.W.; Jin, Y.X.; Zhang, Y.F.; Fen, L.H.; Chen, L.H.; Dong, X.Y. Magneto-optic fiber Sagnac modulator based on magnetic fluids. Opt. Lett. 2011, 36, 1425-1427.

83. Zhao, Y.; Lv, R.Q.; Ying, Y.; Wang, Q. Hollow-core photonic crystal fiber Fabry-Perot sensor for magnetic field measurement based on magnetic fluid. Opt. Laser Technol. 2012, 44, 899-902. 
84. Zu, P.; Chan, C.C.; Lew, W.S.; Jin, Y.X.; Zhang, Y.F.; Liew, H.F.; Chen, L.H.; Wong, W.C.; Dong, X.Y. Magneto-optical fiber sensor based on magnetic fluid. Opt. Lett. 2012, 37, 398-400.

85. Ning, Y.N.; Chu, B.C.B.; Jackson, D.A. Interrogation of a conventional current transformer by a fiberoptic interferometer. Opt. Lett. 1991, 16, 1448-1450.

86. Ning, Y.N.; Liu, T.Y.; Jackson, D.A. Two low-cost robust electrooptic hybrid current sensors capable of operation at extremely high-potential. Rev. Sci. Instru. 1992, 63, 5771-5773.

87. Tonnesen, O.; Beatty, N.; Skilbreid, O. Electrooptic methods for measurement of small DC currents at high-voltage level. IEEE Trans. Power Delivery 1989, 4, 1568-1572.

88. Pilling, N.A.; Holmes, R.; Jones, G.R. Optical-fiber line current measurement system. In Sixth International Conference on Dielectric Materials, Measurements and Applications, Manchester, England, 7 September 1992, 363, 278-281.

(C) 2012 by the authors; licensee MDPI, Basel, Switzerland. This article is an open access article distributed under the terms and conditions of the Creative Commons Attribution license (http://creativecommons.org/licenses/by/3.0/). 